Encyclopedia of Clinical Neuropsychology 
Jeffrey S. Kreutzer - John DeLuca Bruce Caplan

Editors

\section{Encyclopedia of Clinical Neuropsychology}

Second Edition

With 230 Figures and 197 Tables

第 Springer 
Editors

Jeffrey S. Kreutzer

Department of Physical

Medicine and Rehabilitation

Virginia Commonwealth University

Richmond, VA, USA

Bruce Caplan

Independent Practice

Wynnewood, PA, USA

\author{
John DeLuca \\ Kessler Foundation \\ Pleasant Valley Way \\ West Orange, NJ, USA
}

ISBN 978-3-319-57110-2 ISBN 978-3-319-57111-9 (eBook)

ISBN 978-3-319-57112-6 (print and electronic bundle)

https://doi.org/10.1007/978-3-319-57111-9

Library of Congress Control Number: 2018945469

(C) Springer Science+Business Media, LLC 2011

(C) Springer International Publishing AG, part of Springer Nature 2018

This work is subject to copyright. All rights are reserved by the Publisher, whether the whole or part of the material is concerned, specifically the rights of translation, reprinting, reuse of illustrations, recitation, broadcasting, reproduction on microfilms or in any other physical way, and transmission or information storage and retrieval, electronic adaptation, computer software, or by similar or dissimilar methodology now known or hereafter developed.

The use of general descriptive names, registered names, trademarks, service marks, etc. in this publication does not imply, even in the absence of a specific statement, that such names are exempt from the relevant protective laws and regulations and therefore free for general use.

The publisher, the authors, and the editors are safe to assume that the advice and information in this book are believed to be true and accurate at the date of publication. Neither the publisher nor the authors or the editors give a warranty, express or implied, with respect to the material contained herein or for any errors or omissions that may have been made. The publisher remains neutral with regard to jurisdictional claims in published maps and institutional affiliations.

This Springer imprint is published by Springer Nature, under the registered company Springer International Publishing AG

The registered company address is: Gewerbestrasse 11, 6330 Cham, Switzerland 


\section{Preface}

Neuropsychology is a rapidly evolving field with inspiring advances in neurotechnology, assessment, and intervention occurring regularly. An act of labor and love, the conceptualization, compilation, and production of the Encyclopedia of Clinical Neuropsychology (2nd ed.) spanned more than 4 years. Building on the foundation of the first edition, the editors-in-chief sought to provide a current corpus of content of empirically based, relevant, and practical information. To this end, we added three new topical sections: neuroimaging, military neuropsychology, and DSM-5 and enlisted the help of three superb new section editors: Erin Bigler, Ph.D., Risa Nakase-Richardson, Ph.D., and Ana Mills, Psy.D, respectively, to orchestrate the new sections. An immense project, the encyclopedia now contains more than 2,200 entries. More than 1,600 first edition entries were updated and 230 new entries were added. The newest edition of the encyclopedia now reflects the work of nearly 900 authors.

As with the first edition, we have aimed to create a comprehensive reference for professionals involved in the evaluation, diagnosis, and rehabilitation of children and adults with neurological disorders. We have also provided students and researchers with the breadth of knowledge to facilitate success in evaluating and treating these patients. Suggested readings and cross references direct readers to additional information on the various topics. We anticipate that the Encyclopedia of Clinical Neuropsychology will be the first place readers turn for factual, relevant, and comprehensive information.

August 2018

Jeffrey S. Kreutzer

John DeLuca

Bruce Caplan 


\section{Acknowledgment}

We are indebted to our cadre of esteemed Associate Editors for helping to develop their sections, recruit contributors, and ensure the presence of consistently high quality entries. We express our utmost appreciation to the brilliant group of authors whose efforts form the core of our project. We are immensely indebted to the superb Springer Major reference works team including Janice Stern, Michael Hermann, and Sonja Peterson. They taught us, encouraged us, kept us organized and on track, and helped us in every step of the way. We are also grateful to our students, patients and their families from whom we learned much about facing challenges and the value of being practical. 


\section{List of Topics}

\section{Cancer and Tumors-Bruce Caplan}

\section{Section Editor: Carol L. Armstrong}

Acoustic Neuroma

Acute Lymphoblastic Leukemia

Acute Myelogenous Leukemia

Acute Radiation Somnolence

Adenoma

Angioma, Cavernous Angioma

Astrocytoma

Atypical Teratoid/Rhabdoid Tumor (AT/RT)

Biopsy

Brachytherapy

Brain Tumor

Brainstem Glioma

Centigray

Cerebellar Mutism

Chemotherapy

Cognitive Affective Syndrome

Craniopharyngioma

Craniospinal Radiotherapy

Cyst

Desmoplastic Gangliocytoma

Dysembryoplastic Neuroepithelial Tumor

Early-Delayed Effects of Radiation

Endothelial Proliferation

Ependymoma

Ewing's Sarcoma

Fibrillary Astrocytoma

Ganglioglioma

Germ Cell Tumors

Glioblastoma Multiforme

Glioma

Gliomatosis Cerebri
Hamartoma

Hemangioblastoma

Hemangioma

Hemangiopericytoma

Hygroma

Hypothalamic Glioma

Intensity-Modulated Radiation Therapy

Involved Field Radiotherapy

Late-Delayed Effects of Radiation Therapy

Late Effects of Radiation Therapy

Leukemia

Lymphoplasmacytic Tumor

Meninges Type Tumors

Meningioma

Metastasis

Neoplasms

Neuroblastoma

Neurocytoma

Neurofibromatosis Type 1 (NF1)

Neurofibromatosis Type 2 (NF2)

Neuroma

Oligoastrocytoma

Oligodendroglioma

Optic Glioma, Optic Pathway Glioma

Pilocytic Astrocytoma and Juvenile Pilocytic

Astrocytoma

Pineal Tumors

Pituitary Adenoma

Pituitary Mass

Plasmacytoma

Pleomorphic, Pleomorphism

Plexiform Neurofibroma

Posterior Fossa

Posterior Fossa Syndrome 
Primitive Neuroectodermal Tumor

Proton Beam Therapy

Radiation Injury

Radiation Necrosis

Radiation Oncology

Radiosurgery, Stereotactic Radiosurgery

Retinoblastoma

Sarcoma

Schwannoma

Stereotactic Radiation Therapy

Tectal Glioma

Teratoma

Tuberous Sclerosis

Tumor Grade

Xanthoastrocytoma

Bacterial Ventriculitis

Chemo Brain

Dysmetria

Epigenetics

Eyeblink Conditioning

Intraoperative fMRI

Neuroinformatics

Pseudomeningocele

Teleneuropsychology

Transcranial Electrical Stimulation (tES)

\section{Forensics-Jeff Kreutzer}

\section{Section Editor: Julie K. Lynch}

AACN Practice Guidelines

Actus Reus

Admissibility

Aggravating Factors

Americans with Disabilities Act of 1990

Arbitration

Atkins v. Virginia

Attorney

Barefoot v. Estelle (1983)

Baxter v. Temple (2005)

Beyond a Reasonable Doubt

Bias

Burden of Proof

Capacity

Chandler Exterminators v. Morris (1992)

Chapple v. Ganger

Civil Litigation

Clear and Convincing Evidence
Competency

Confidentiality

Criminal Forensics

Criminal Litigation

Criminal Responsibility

Cross-Examination

Daubert v. Merrell Dow Pharmaceuticals (1993)

Death Penalty

Deposition

Detroit Edison v. NLRB (1979)

Diminished Capacity

Diminished Responsibility

Direct Examination

Discovery

Dissimulation

Due Process

Dusky v. United States (1960)

Estelle v. Smith (1981)

Ethical Principles in Forensics

Expert v. Treater Role

Expert Witness

Fake Bad Scale

Federal Rules of Civil Procedure 26 and 35

Federal Rules of Criminal Procedures

Emphasizing 12.2

Federal Rules of Evidence

Financial Capacity

Ford v. Wainwright

Forensic Neuropsychologist

Forensic Neuropsychology

Forensic Psychology

FRE 104, 403, 702, and 704

Frontal Lobe Defense

Furman v. Georgia (1972)

Guilty But Mentally III

Hare Psychopathy Checklist

Hearsay Evidence

In Camera Review

Independent Medical Evaluation

Independent Neuropsychological Examination

Informed Consent

Insanity

Insanity Defense

Intent, General v. Specific

Irresistible Impulse

Jenkins v. U.S. (1962)

Joiner v. General Electric (1997)

Kumho Tire v. Carmichael 
Learned Treatise

Legal Competency

Litigation

Lockett v. Ohio (1978)

M'Naghten Rule

Malingering

Mediation

Medical Malpractice

Mens Rea

Mental State at Offense

Miranda v. Arizona

Mitigating Factors

Motion for Summary Judgment

Motion in Limine

Penry v. Lynaugh (1989)

Personal Injury

Preponderance of the Evidence

Prima Facie Case

Privilege

Proximate Cause

Section 504 of the Rehabilitation Act of 1973

Self-Incrimination

Specialty Guidelines for Forensic Psychology

Structured Interview of Reported Symptoms (SIRS)

Subpoena

Subpoena Duces Tecum

Testamentary Capacity

Testifying Expert Versus Fact Witness

Third Party Observer

Trial Consulting

Voir Dire

Workers' Compensation

\section{Pediatrics-Bruce Caplan}

\section{Section Editor: Jennifer Ann Niskala Apps}

504 Plan

AAMD Adaptive Behavior Scales

Absence Epilepsy

Accommodations

Adaptive Behavior Assessment System: Third

Edition

Anencephaly

Angelman Syndrome

Applied Behavior Analysis

Asperger's Disorder
Assistive Technology

Attention Deficit Hyperactivity Disorder

Augmentative and Alternative Communication (AAC)

Autism Diagnostic Interview, Revised

Autism Diagnostic Observation Schedule

Autistic Disorder

Bayley Scales of Infant and Toddler Development

Beery Developmental Test of Visual-Motor Integration (VMI), Sixth Edition

BASC-3

Rolandic Epilepsy

California Verbal Learning Test - Children's Version

Central Auditory Processing Disorder

Cerebral Palsy

Achenbach System of Empirically Based Assessment (ASEBA)

Childhood Autism Rating Scales

Children's Category Test

Children's Memory Scale

Conners 3rd Edition (Conners 3; Conners 2008)

Conners Comprehensive Behavior Rating Scales ${ }^{\mathrm{TM}}$

Conners' Continuous Performance Test Third Edition

Delis-Kaplan Executive Functioning System

Developmental Gerstmann Syndrome

Differential Ability Scales (DAS and DAS-II)

Down Syndrome

Dyslexia

Expressive One-Word Picture Vocabulary Test

Family-Centered Care

Febrile Seizures

Fetal Alcohol Spectrum Disorder

Fragile X Syndrome

Functional Independence Measure for Children

General Language Composite

Heterotopia

Human Figure Drawing Tests

Hyperlexia

Individuals with Disabilities Education Act

Intellectual Disability

Juvenile Huntington Disease

Juvenile Myoclonic Epilepsy

Juvenile Parkinson's Disease

Landau-Kleffner Syndrome

Learning Disability 
Leiter International Performance Scale, Third Edition

Lennox-Gastaut Syndrome

Megalencephaly

Microcephaly

Minimal Brain Dysfunction

Modified Checklist for Autism in Toddlers, Revised, with Follow-Up (M-CHAT-R/F), also M-CHAT

Mullen Scales of Early Learning

Myelomeningocele

Myoclonic Epilepsy in Infancy

National Dissemination Center for Children with Disabilities

National Organization for Rare Disorders (NORD)

NEPSY-II

No Child Left Behind Act of 2001

Nonverbal Learning Disabilities

Peabody Individual Achievement Test, Revised

Peabody Picture Vocabulary Test

Perceptual Reasoning Index

Periventricular Leukomalacia

Personality Inventory for Children

Pervasive Developmental Disorder NOS

Phenylketonuria

Plasticity

Prader-Willi Syndrome

Prematurity and Low Birth Weight

Processing Speed Index (WPPSI-IV)

Public Law 94-142

Semantic Pragmatic Disorder (SPD)

Sensitive Periods

Abusive Head Trauma

Soft Signs

Stanford-Binet Intelligence Scales and Revised Versions

Stroop Color and Word Test, Children's Version TEACCH

Tourette Syndrome

Turner Syndrome

Vineland Adaptive Behavior Scales

Vineland Social Maturity Scales

Waisman Center, University of WisconsinMadison

Wechsler Intelligence Scale for Children

Wechsler Preschool and Primary Scale of Intelligence
West Syndrome

Williams Syndrome

Continuous Auditory Test of Attention

(CATA)

Duplication 7 Syndrome

Movement-Jeff Kreutzer

Section Editor: Douglas I. Katz

Abasia

Action Tremor

Akathisia

Akinesis

Allokinesia

Apraxia

Astasia-Abasia

Ataxia

Automatism

Balance Disorders

Bell's Palsy

Bradykinesia

Chorea

Circumduction

Dopamine-Related Dyskinesia

Dysdiadochokinesia

Dystonia

Essential Tremor

Festination

Gait Disorders

Headache

Hemiparesis

Hemiplegia

Homunculus

Hypertrophy

Intention Tremors

Internal Capsule

Internuclear Ophthalmoplegia

Lateral Gaze Palsy

Leukodystophy

Locked-In Syndrome

Magnetic Gait

Masked Facies

Ménière's Syndrome

Micrographia

Motor Stereotypy

Movement Disorders

Myoclonus 
Orthostatic Tremor

Parkinson Plus Syndromes

Parkinsonism

Physiologic Tremor

Ptosis

Restless Leg Syndrome

Retropulsion

Rigidity

Spastic Gait

Tardive Dyskinesia

Thalamus

Tic

Tremor

Wilson's Disease

\section{Medical-Jeff Kreutzer}

\section{Section Editor: Nathan D. Zasler}

Abbreviated Injury Scale

Ablation

Alpha Rhythm

Angiography, Cerebral

Babinski Reflex

Blood Alcohol Level

Blood Flow Studies

Blood Oxygen Level Dependent (BOLD)

Brainstem Auditory Evoked Responses

Carotid Angiography

Carotid Endarterectomy

Commissurotomy

Computed Tomography

Coronary Artery Bypass Graft

Cranioplasty

Craniotomy

Deep Brain Stimulator (Parkinson's)

Diffusion Tensor Imaging

Digital Subtraction Angiography

Doppler Ultrasound

Dysconjugate Gaze

Electrodiagnosis

Electroencephalography

Endotracheal Tube

Event-Related Potentials

Evidence-Based Practice

Evoked Potentials

Fixed Pupils
Fluorodeoxyglucose Positron Emission

Tomography

Fractional Anisotropy

Frontal Lobotomy

Functional Imaging

Gamma Knife

Hemispherectomy

Hemodynamic Response

Hemoglobin

International Classification of Diseases

Intracarotid Sodium Amobarbital Test

Leukotomy

Lobectomy

Lumbar Puncture

Luxury Perfusion

Magnetic Resonance Angiography

Magnetic Resonance Imaging

Magnetic Resonance Spectroscopy

Magnetoencephalography

Midline Shift

Neuroimaging

Neurologic Examination

Nuclear Magnetic Resonance

Olfaction

P300

Pallidotomy

PET

Physiatric Assessment

Pneumoencephalography

Polysomnography

Post-traumatic Headache

Posturography

Prefrontal Lobotomy

Quantitative Encephalography

Radiotherapy

Rancho Los Amigos Scale

Regional Cerebral Blood Flow

Sensorimotor Assessment

Shunts

Signs

Single Photon Emission Computed Tomography

Somatosensory Evoked Potentials

Stereotaxic Surgery

Temporal Lobectomy

Tesla

Transcranial Doppler

Transcranial Magnetic Stimulation

UBO 
Ventriculostomy

Visual Evoked Potentials

Voxel

Wada Test

\section{Academic Evaluation-John Deluca}

\section{Section Editor: Rik Carl D'Amato}

\author{
Abstract Reasoning \\ Academic Competency \\ Academic Skills \\ Acalculia \\ Arithmetic Reasoning \\ Auditory Discrimination \\ Auditory Processing \\ Social Awareness
}

Basic Achievement Skills Inventory

Bender Visual-Motor Gestalt Test II

Cognitive Assessment System

Compensatory Education Approach

Constructional Apraxia

Deaf/Hearing Impairment

Dean-Woodcock Neuropsychological

Assessment System

Developmental Milestones, Stages

Dyscalculia

Educational Testing

Emotional Disturbance

Emotional Intelligence

Emotionality

Executive Functioning

Finger Localization

Individual Education Program

Mainstreaming

Manual Dexterity

Manual Strength

McCarthy Scales of Children's Abilities

Mental Tracking

Metacognition

Multitasking

National Adult Reading Test and Orientation (Left-Right)

Orientation

Orientation (Left-Right)

Other Health Impairment

Otis-Lennon School Ability Test

Phonemic Awareness
Phonics

Physical Disabilities

Praxis

Primary Handicapping Conditions

Problem Solving

Proprioception

Rapid Automatic Naming

Reading

Reading Comprehension

Reading Fluency

Reality Orientation

Reasoning

Remedial Education Approach

Response to Intervention

Scholastic Aptitude Test

Secondary Handicapping Condition

Self-Regulation

Sequential Processing

Simultaneous Processing

Social Competence

Special Education

Speech/Communication Disabilities

Stereognosis

Strength-Based Education

Tests of General Educational Development

Vocabulary

Wechsler Individual Achievement Test

Wechsler Test of Adult Reading

Woodcock-Johnson IV

Progress Monitoring

Neuropsychologically Driven Interventions

Evidence-Based Interventions

Single-Case Design Interventions

Inclusive Education

Homeschooling (Homeschool

Education)

Charter School

Neurodiversity

Neuroeducation

Neuroleadership

Neurotechnology

Neurosystems

Immigrant Languages

Psychoeducation

Education

Prevention

Family Education

Neurotherapy 
Neurofeedback

Mindfulness

Infant/Toddler Preschool Assessment (0-6)

\section{Attention and Concentration-John Deluca}

Section Editor: Ronald Cohen and Adam J. Woods

Action-Intentional Disorders

Anosognosia

Anterior Cingulate Cortex

Arousal

Attention

Attention Network Test (ANT)

Automaticity

California Computerized Assessment Package

Cancellation Tests

Capacity Limitations

Catatonic Behavior

Confabulation

Conners Rating Scales

Consciousness

Continuous Performance Tests

Controlled Attention

Cue Dominance

Default Network

Delirium

Delusion

Dichotic Listening

Digit Span

Digit Symbol Substitution Test

Directed Attention

Discriminability

Disengagement of Attention

Disinhibition

Divided Attention

Effort

Enhancement

Extinction

Facilitation

Filtering

Focused Attention

Habituation

Hemiinattention

Hemikinesis

Inferior Parietal Lobule

Information Processing Speed
Inspection Time

Interference

Lethargy

N-Back Paradigm

Orienting Response

Paced Auditory Serial Attention Test

Parallel Processing

Processing Speed Index

Searchlight Hypothesis

Selective Attention Models

Serial Sevens

Serial/Sequential Processing

Signal Detection Theory

Span of Apprehension

Sustained Attention

Symbol Search

Test of Everyday Attention

Test of Everyday Attention for Children

Thalamic Gating

Vigilance

Working Memory

Yerkes-Dodson Law

Attentional Response Bias

Eye Tracking

Flanker Task

D2 Test of Attention

Digit Vigilance Test

Brief Test of Attention

Tests of Variables of Attention

Auditory Selective Attention Test

Visual Search and Attention Test

Attentional Blink

Vertical Neglect

Useful Field of View (UFOV)

Behavioral Inattention Test (BIT)

Visual Search

Troxler Fading

Priming

Pre-Pulse Inhibition

Inattentional Blindness

\section{Brain Injury-Jeff Kreutzer}

Section Editor: James F. Malec

Acceleration Injury

Acute Respiratory Distress Syndrome

Appalic Syndrome 
Automated Neuropsychological Assessment Metrics

Battle's Sign

Biomechanics of Injury

Blast Injury

Brain Swelling

Coma

Concussion

Contrecoup Injury

Cortical Contusion

Craniectomy

Deceleration Injury

Decerebrate Posturing

Decorticate Posturing

Depressed Skull Fracture

Diffuse Axonal Injury

Distractibility

Edema

Electrical Brain Injury

Embolism

Epidemiology

Epidural Hematoma

Fatigue

Focal Lesion, Contusion

Head Injury

Heterotopic Ossification

Holistic Brain Injury Rehabilitation

Hypersomnia

Hypoarousal

Immediate Post-Concussion Assessment and

Cognitive Testing

Impaired Self-Awareness

Initiation

Insomnia

Lead Exposure

Loss of Consciousness

Mass Effect

Memory Impairment

Mercury Exposure

Mild Traumatic Brain Injury

Minimally Conscious State

Minimally Responsive State

Moderate Brain Injury

Narcolepsy

Obstructive Sleep Apnea Syndrome

Occult Head Injury

Outcome, Outcome Measurement

Pediatric Traumatic Brain Injury
Penetrating TBI

Pneumothorax

Polytrauma

Postacute Brain Injury Rehabilitation

Postconcussion Syndrome

Post-traumatic Confusional State

Pupillary Light Response, Pupillary Response

Raccoon Eyes

Rotational Acceleration

Secondary Brain Injury

Severe Brain Injury

Shearing Injury, Shear Strain

Skull Fracture

Sleep Apnea Syndrome

Sleep Disturbance

Spinal Cord Injury

Sport-Related Concussion

Stupor

Subgaleal Hematoma

Time to Follow Commands

Toxic Exposure

Trauma Center

Traumatic Brain Injury (TBI)

Traumatic Brain Injury Model System

Vegetative State (Persistent)

Whiplash

Ohio State University Traumatic Brain Injury Identification Method

Normal Pressure Hydrocephalus

\section{CNS-John Deluca}

Section Editor: Randall E. Merchant and John DeLuca

Afferent

Alzheimer's Disease

Amygdala

Arcuate Fasciculus

Associational Fibers

Auditory Pathway

Autonomic Nervous System

Basal Forebrain

Basal Ganglia

Blood-Brain Barrier

Brain Death

Brodmann's Areas of the Cortex

Caudate Nucleus 
Cerebellum

Cerebral Cortex

Cerebral Edema

Cerebral Perfusion Pressure

Commissures, Cerebral

Corpus Callosum

Cortical Motor Pathways

Cortical-Subcortical Loop

Cranial Nerves

Dentate Gyrus

Diencephalon

Efferent

Visual Fields

Forebrain

Fornix

Frontal Lobe

Ganglion

Globus Pallidus

Gray Matter

Hemi-attention Syndrome

Hindbrain

Hydrocephalus

Insular Lobe

Intracranial Pressure

Korsakoff's Syndrome

Limbic System

Localization

Locus Ceruleus

Mediodorsal Nucleus of Thalamus

Medulla

Medulloblastoma

Meninges

Mesolimbic Dopaminergic Projections

Midbrain

Midbrain Raphe

Multiple Sclerosis

Myelin

Neocortex

Neuroglia

Neuron

Nucleus Accumbens

Nucleus Basalis of Meynert

Occipital Lobe

Pallidum

Papez Circuit

Parietal Lobe

Parkinson's Disease

Periventricular White Matter
Pneumocephalus

Pons

Postcentral Gyrus

Precentral Gyrus

Putamen

Pyramidal System

Raphe Nucleus

Reticular Activating System

Rostral

Somatosensory System

Spinal Cord

Striate Cortex

Striatum

Subfornical Organ

Subiculum

Substantia Nigra

Superior Colliculus

Superior Longitudinal Fasciculus

Supramarginal Gyrus

Sylvian Fissure

Temporal Lobe

Uncus

Ventral Tegmental Area of Midbrain

Ventricles

Ventricular Enlargement

Vestibular Nuclei

Visual Cortex

White Matter

Caudal

Schizophrenia

\section{Cognitive Rehabilitation-John Deluca}

Section Editor: Sarah A. Raskin

Active Limb Activation

Activities of Daily Living (ADL)

Attention Training

Cognitive Correctors

Cognitive Functioning

Cognitive Rehabilitation

Compensatory Strategies

Concept Learning

Constraint Induced Therapy

Error Recognition and Correction

Errorless Learning

Insight: Effects on Rehabilitation

Lighthouse Technique 
Memory Book

Method of Vanishing Cues

Mnemonic Techniques

Neuropsychological Rehabilitation

Process Training

Prosthetic Memory Aids

Quality of Life

Strategy Substitution

Verbal Mediation

Computerized Treatment

Goal Management Training

Academic Techniques

Brain Training

\section{Dementia-John Deluca}

\section{Section Editor: Gordon Chelune}

Alzheimer's Dementia

Amyloid Plaques

Amyotrophic Lateral Sclerosis

Apoptosis

Atrophy

Beta-Interferons

Binswanger's Disease

Blessed Dementia Scale

CADASIL

Cholinesterase Inhibitors

Clinical Dementia Rating

Consortium to Establish a Registry on

Alzheimer's Disease

Corticobasal Degeneration

Dementia Rating Scale-2

Dementia with Lewy Bodies

Demyelination

Encephalopathy

Frontal Temporal Dementia

Frontotemporal Lobar Degenerations

Gliosis

Huntington's Disease

Hypertensive Encephalopathy

Leukoaraiosis

Lewy Bodies

Logopenic Aphasia

Metabolic Encephalopathy

Mild Cognitive Impairment

Modified Mini-Mental State Examination

Multi-infarct Dementia
Neurofibrillary Tangles

Optic Neuropathy

Paralysis Agitans

Parkinson's Dementia

Pick's Disease

Primary Progressive Multiple Sclerosis

Progressive Aphasia

Progressive Supranuclear Palsy

Relapsing-Remitting Multiple Sclerosis

Secondary-Progressive Multiple Sclerosis

Semantic Dementia

Senile Dementia

Senile Plaques

Small Vessel Ischemic Disease

Tauopathy

Vascular Cognitive Impairment

Vascular Dementia

\section{Frontal Lobe-John Deluca}

Section Editor: Paul Malloy

Abulia

Adynamia

Akinetic Mutism

Alien Hand Syndrome

Anosmia

Apathy

Avolition

Behavior Rating Inventory for Executive Function

Category Test

Central Executive

Cingulate Gyrus

Cingulum

Delayed Response Tasks

Design Fluency Test

Dorsolateral Prefrontal Cortex

Environmental Dependency

Executive Abilities: Methods and Instruments for Neurobehavioral Evaluation and Research

Executive Interview

Figural Fluency Test

Frontal Systems Behavior Scale

Gegenhalten

Glabellar Reflex

Grasp Reflex

Klüver-Bucy Syndrome 
Motor Impersistence

Palmomental Reflex

Perseveration

Prefrontal Cortex

Reduplicative Paramnesia

Rooting Reflex

Ruff 2 and 7 Selective Attention Test

Ruff Neurobehavioral Inventory

Ruff-Light Trail Learning Test

Sexual Disinhibition

Snout Reflex

Stroop Effect

Suck Reflex

Utilization Behavior

Witzelsucht

Iowa Scales of Personality Change

\section{Functional Evaluation-Bruce Caplan}

\section{Section Editor: Tamara Bushnik}

Activities of Daily Living Questionnaire

Alzheimer's Disease Cooperative Study ADL

Scale

Amyotrophic Lateral Sclerosis Functional Rating

Scale

Ashworth Spasticity Scale (and Modified

Version)

ASIA Impairment Scale

Assessment of Life Habits (LIFE-H)

Assessment of Motor Process Skills

Barthel Index

Berg Balance Scale

Bristol Activities of Daily Living Scale

Brunel Balance Assessment

CHART Short Form

Cognitive Log

Community Integration Questionnaire

Craig Handicap Assessment and Reporting

Technique

Direct Assessment of Functional Status

Disability Assessment for Dementia

Disability Rating Scale

Driving Assessment

EuroQol/EQ-5D

Expanded Disability Status Scale

Fibromyalgia Impact Questionnaire

Frenchay Activity Index
Fugl-Meyer Assessment of Sensorimotor Impairment

Functional Ambulation Classification

Functional Assessment

Functional Assessment Measure

Functional Assessment Multiple Sclerosis

Functional Autonomy Measurement System

Functional Capacity Evaluations

Functional Independence Measure

Functional Status

Functional Status Questionnaire

General Well-Being Schedule

Glasgow Coma Scale

Glasgow Outcome Scale

Glasgow Outcome Scale: Extended

Health Assessment Questionnaire

High-Level Mobility Assessment Test

Impact on Participation and Autonomy

Questionnaire

Independent Living Scales ${ }^{\circledR}$

Informant Questionnaire on Cognitive Decline in the Elderly

Instrumental Activities of Daily Living

Jebsen-Taylor Hand Function Test

Katz Index of ADLs

Kohlman Evaluation of Living Skills

Late Life Function and Disability Index

Lawton-Brody Instrumental Activities of Daily Living Scale

Loewenstein Occupational Therapy Cognitive Assessment

Mayo-Portland Adaptability Inventory

Mobility, Self-Care, and Domestic Life (MOSES)

Motor Activity Log

Motor Assessment Scale

Multiple Sclerosis Quality of Life-54

Questionnaire

National Institutes of Health Stroke Scale

Norwegian Function Assessment Scale

Nottingham Health Profile

Older Americans' Resources and Services

Multidimensional Functional Assessment

Questionnaire

Participation Measure for Post-acute Care

Participation Objective, Participation Subjective

Patient Competency Rating Scale

Patient Generated Index

Performance-Oriented Mobility Assessment 
Physical Functional Performance

PM-PAC Computerized Adaptive Testing

Postural Assessment Scale for Stroke

Postural Control and Balance for Stroke

Profile of Functional Impairment in

Communication

Psychological Health Assessment Questionnaire

Rankin Scale

Raskin Rating Scale

Reintegration to Normal Living Index

Rivermead Mobility Index

Roland-Morris Questionnaire

Severe Impairment Battery

SF-36/SF-12

Short Physical Performance Battery

Sickness Impact Profile

6-Minute Walk Test

Spinal Cord Independence Measure (SCIM)

Stroke Activity Scale

Stroke Impact Scale

Stroke-Adapted Sickness Impact Profile

Supervision Rating Scale

Timed Up and Go Test

UCSD Performance-Based Skills Assessment

Unified Parkinson's Disease Rating Scale

Visual Analog Scale

Wolf Motor Function Test

PART-O

\section{General/Historical-Bruce Caplan}

\section{Section Editor: Anthony Y. Stringer}

Akelaitis, Andrew John Edward (“A.J.”)

$$
\text { (1904-1955) }
$$

Alzheimer, Alois (1864-1915)

American Academy of Clinical Neuropsychology (AACN)

American Academy of Neurology (AAN)

American Board of Pediatric Neuropsychology

American Board of Professional Psychology (ABPP)

American Board of Professional

Neuropsychology (ABN)

American Board of Rehabilitation Psychology

American College of Professional

Neuropsychology (ACPN)

American Congress of Rehabilitation Medicine
American Psychological Association (APA)

American Psychological Association (APA), Division 22

American Psychological Association (APA), Division 40

Association for Postdoctoral Programs in

Clinical Neuropsychology (APPCN)

Babcock, Harriet (1807-1952)

Balint, R. (Rezso (Rudolf) Balint) (1874-1929)

Behaviorism

Benton, Arthur (1909-2006)

Ben-Yishay, Yehuda (1933-)

Brain Injury Association of America

Broca, Pierre Paul (1824-1880)

Butters, Nelson (1937-1995)

Center for Outcome Measurement in Brain Injury (COMBI)

Centers for Medicare and Medicaid Service

Christensen, Anne-Lise (1926- )

Clinical Neuropsychology

Commission on Accreditation of Rehabilitation Facilities (CARF)

Cultural Diversity in Neuropsychology

Damasio, Antonio R. (1944-)

De Renzi, Ennio (1924-)

Dejerine, Joseph Jules (1849-1917)

Diller, Leonard (1924- )

Ethics in the Practice of Clinical

Neuropsychology: Foundations and

New Horizons

Gage, Phineas (1823-1860)

Gazzaniga, M. S. (1939-)

Gerstmann, Josef (1887-1969)

Geschwind, Norman (1926-1984)

Goldman-Rakic, Patricia (1937-2003)

Goldstein, Kurt (1878-1965)

Goodglass, Harold (1920-2002)

H.M.; Also the Case of H.M., Molaison, Henry (1926-2008)

Halstead, Ward (1908-1968)

Hebb, Donald (1904-1985)

Hécaen, Henry (1912-1983)

Heilman, Kenneth M. (1938-)

International Neuropsychological Society

International Neuropsychological

Symposium

Jennett, Bryan (1926-2008)

Kaplan, Edith (1924-2009) 
Kinsbourne, Marcel (1931-)

Korsakoff (Korsakov), Sergei Sergeievich (1854-1900)

Lashley, Karl Spencer (1890-1958)

Lezak, Muriel

Liepmann, Hugo Karl (1863-1925)

Luria, Alexander Romanovich (1902-1977)

Meier, Manfred John (1929-2006)

Milner, Brenda Atkinson (1918-)

Mishkin, Mortimer (1926-)

Monrad-Krohn, Georg H (1884-1964)

National Academy of Neuropsychology (NAN)

National Center for the Dissemination of

Disability Research (NCDDR)

National Institute of Neurological Disorders and Stroke

National Institute on Disability Independent

Living and Rehabilitation Research

(NIDILRR)

National Institutes of Health (NIH)

National Rehabilitation Information Center

(NARIC)

Neuropsychiatry

Neuropsychology

Occupational Therapy

Parsons, Oscar (1920-2000)

Penfield, Wilder (1891-1976)

Physiatry

Pribram, Karl H. (1919-)

Rehabilitation Psychology

Reitan, Ralph (1922-)

Rosenthal, Mitchell (1949-2007)

Rourke, Byron (1939-)

Sperry, Roger Wolcott (1913-1994)

Tan

Teuber, Hans-Lukas (1916-1977)

Warrington, Elizabeth

Wernicke, Karl (1848-1905)

Zangwill, Oliver (1913-1987)

American Board of Clinical Neuropsychology $(\mathrm{ABCN})$

Diagnostic and Statistical Manual of Mental

Disorders (DSM)

Hispanic Neuropsychological Society

Hispanic/Latino Neuropsychology

Neuropsychology in Africa

Houston Conference

Gerald Goldstein

\section{Genetics}

Section Editor: John DeLuca

Adoption Studies

Allele

Chromosome

Cognitive Processing

Deoxyribonucleic Acid (DNA)

Frontal Lobe Syndrome

Gene

Gene-Environment Interaction

Genotype

Heritability

Mesial Frontal System

Orbitofrontal System

Phenotype

Polymorphism

Tower of London

Transgenic

Twin Studies

\section{Illness or Disease-John Deluca}

Section Editor: Susan K. Johnson

Acquired Immunodeficiency Syndrome (AIDS)

Arachnoid Cyst

Brain Abscesses

Carbon Monoxide Poisoning

Chelation

Chronic Fatigue Syndrome

Chronic Obstructive Pulmonary Disease

Circadian Rhythms

Creutzfeldt-Jakob Disease

Cushing's Syndrome

Developmental Delay

Diabetes Mellitus

Encephalitis (Viral)

Epstein-Barr Virus

Fibromyalgia

Guillain-Barré Syndrome

Gulf War Syndrome

Herniation Syndromes

Herpes Simplex Encephalitis

Hypoglycemia

Hypothyroidism

Iatrogenic

Inflammation 
Kuru

Lyme Disease

Meningitis

Multiple Chemical Sensitivity

Myasthenia Gravis

Neurasthenia

Neuropathy

Neurosyphilis

Neurotoxins

Organic Brain Syndrome

Prion Disease

Psychogenic Nonepileptic Seizures

Second Impact Syndrome

Sick Building Syndrome

Sjogren's Syndrome

Symptom

Syndrome

Systemic Lupus Eythematosus

Temporomandibular Joint Dysfunction

Toxic-Metabolic Encephalopathy

Unexplained Illness

Wallenberg's Syndrome

\section{Intelligence-John Deluca}

Section Editor: Nancy D. Chiaravalloti

Advanced Progressive Matrices

Colored Progressive Matrices

Estimation Methods

Flynn Effect

Full Scale IQ

Intelligence

Intelligence Quotient

Kaufman Adult Intelligence Test

Kaufman Brief Intelligence Test

Mental Age

Perceptual Organization Index

Performance IQ

Porteus Maze

Premorbid Intelligence

Raven's Progressive Matrices

Shipley Institute of Living Scale

Standard Progressive Matrices

Verbal Comprehension Index

Verbal IQ

Wechsler Intelligence Tests for Adults and

Children
Working Memory Index

Fluid Reasoning

Visual Spatial Index

\section{Interventions-Jeff Kreutzer}

Section Editor: Bruce Rybarczyk

Activity Restrictions, Limitations

Advocacy

Assisted Living

Attendant Care

Case Management

Circles of Support

Cognitive Behavioral Couples Therapy

Cognitive Behavioral Therapy

Community Re-entry

Community-Based Rehabilitation

Crisis Intervention

Customized Job Retention Services

Discharge Planning

Employment Specialist

Environmental Modifications

Family Team Conference

Family Therapy

Functional Compensation

Group Therapy

Homework

Independent Living Centers

Interdisciplinary Team Rehabilitation

Job Advocacy

Job Analysis

Job Placement

Job Retention

Job Training

Jobsite Training

Life Care Planning

Managed Care

Motivational Interviewing

Pastoral Counseling

Patient-Family Education

Physical Therapy

Problem-Solving Training

Psychotherapy

Recommendation

Recreational Therapy

Rehabilitation Counseling

Relaxation Training 
Sexual Surrogate Therapy

Sheltered Employment

Social and Occupational Functioning Assessment Scale (SOFAS)

Stress Management

Supported Employment

Task Analysis

Transitional Living

Transitional Planning

Vocational Rehabilitation

Cognitive Behavioral Therapy for Insomnia

\section{Language-Bruce Caplan}

Section Editor: Roberta DePompei and Aimee Dietz

Agnosia

Agrammatism

Agraphia

Alexia

American Speech-Language-Hearing Association (ASHA)

American Speech-Language-Hearing Association

Functional Assessment of Communication

Skills for Adults

Anarthria

Anomia

Anomic Aphasia

Aphasia

Aphasia Diagnostic Profiles

Aphasia Tests

Aphonia

Apraxia of Speech

Aprosodia

Articulation

Articulation Disorders

Automatic Language

Bilingual Aphasia

Boston Diagnostic Aphasia Examination

Boston Naming Test

Broca's Aphasia

Circumlocution

Cognitive-Communication Disorder

Communication Ability

Conduction Aphasia

Confrontation Naming

Controlled Oral Word Association Test
Crossed Aphasia

Cue

Discourse Assessment

Dysarthria

Dysphagia

Dysphonia

F-A-S Test

Fluent Aphasia

Global Aphasia

Grammar

Grapheme

Jargon

Language

Lexicon

Literal Paraphasia

Logorrhea

Melodic Intonation Therapy

Metacognitive Skills

Metalinguistic Skills

Morpheme

Multilingual Aphasia Examination

Naming

Neologism

Nonfluent Aphasia

Paragrammatism

Paragraphia

Paralexia

Paraphasia

Phoneme

Phonemic Cue

Phonological Disorder

Phonology

Phrase Length

Pragmatic Communication

Primary Progressive Aphasia

Prosody

Pure Word Deafness

Repetition

Semantic Cue

Semantic Paraphasia

Speech

Speech Sound Disorder

Speech-Language Pathology

Speech-Language Therapy

Stereotypy

Stuttering, Acquired

Stuttering, Developmental

Subcortical Aphasia 
Syntax

Telegraphic Speech

Token Test

Transcortical Motor Aphasia

Transcortical Sensory Aphasia

Verbal Fluency

Wernicke's Aphasia

Wernicke-Lichtheim Model of Aphasia

Western Aphasia Battery

Word Finding

Writing

Perseverative Speech

Gist

Strategic Learning

Semantics

AphasiaBank/TalkBank

Auditory Comprehension

SFA

Conversational Treatments

Learned Non-use

Right-Hemisphere Processing

Social Communication

Pediatric Test of Brain Injury (PTBI)

Reading Comprehension Battery for Aphasia-2

Cognitive Linguistic Quick Test

VNeST

ASHA Quality of Communication

Life Scale

\section{Laterality-Bruce Caplan}

\section{Section Editor: John E. Mendoza and Bruce Caplan}

Agenesis of Corpus Callosum

Ambidexterity

Anomalous Dominance

Anterior Commissure

Association Pathways

Asymmetry

Disconnection Syndrome

Dominance (Cerebral)

Edinburgh Handedness Inventory

Handedness

Hemispheric Specialization

Hemisphericity

Projection Pathways
Septum Pellucidum

Splenium

Split-Brain

Spontaneous Recovery

\section{Memory-John Deluca}

\section{Section Editor: Sarah A. Raskin}

Amnesia

Amnestic Disorder

Amnestic Syndromes

Anterograde Amnesia

Auditory Verbal Learning

Autobiographical Memory

Benign Senescent Forgetfulness

Benton Visual Retention Test

California Verbal Learning Test (California Verbal Learning Test-II)

Cholinergic System

Cognitive Reserve

Consolidation

Cued Recall

Declarative Memory

Dementia

Disorientation

Dissociative Amnesia

Echoic Memory

Episodic Memory

False Memory

Forgetting

Free Recall

Hippocampus

Hopkins Verbal Learning Test

Hypothalamus

Implicit Memory

Incidental Memory

Intrusion Errors

Jamais Vu

Learning

Mammillary Bodies

Medial Temporal Lobe

Memory

Memory for Intentions

$N$-Acetyl Aspartate

Paired-Associate Learning

Post-traumatic Amnesia

Practice Effects 
Presenile Dementia

Primacy Effect

Proactive Interference

Procedural Memory

Prospective Memory

Pseudodementia

Recency Effect

Recent Memory

Recognition Memory Test

Remote Memory

Retrieval Techniques

Retroactive Interference

Retrograde Amnesia

Rey Auditory Verbal Learning Test, Rey AVLT

Rivermead Behavioral Memory Test

Selective Reminding Test

Semantic Memory

Serial Recall

Short-Term Memory

Source Memory

Subcortical Dementia

Transient Global Amnesia

Warrington Recognition Memory Test

Wechsler Memory Scale All Versions

Wernicke-Korsakoff Syndrome

Wide Range Assessment of Memory and Learning

Serial Position Effect

Memory for Intentions Screening Test

\section{Miscellaneous-Jeff Kreutzer}

Section Editor: Stephanie A. KolakowskyHayner

Anticholinergic

Anticonvulsants

Antihistamines

Antihypertensives

Antipsychotics

Anxiolytics

Autoreceptor

Behavior Modification

Compensation Neurosis

Corticotropin-Releasing Hormone

Dysexecutive Index (DEX)

Dysexecutive Syndrome
Dysphoria

Frontal Behavioral Inventory

Frontal Eye Fields

Glucocorticoids

Hormones

Hyperbaric Therapy

Impersistence

Impulsivity

Inhibition

Irritability

Job Restructuring

Neuroleptic Malignant Syndrome

Neuroleptics

Pharmacodynamics

Pharmacokinetics

Phenobarbital

Projective Technique

Psychopharmacology

Psychotropic

Qualitative Data

Qualitative Neuropsychological Assessment

Reuptake

Reuptake Inhibition

Route Finding

Sedative Hypnotic Drugs

Social Skills Training

Steroids

Stimulants

Stimulus-Bound Behavior

Substance Abuse

Supplementary Motor Area (SMA)

Symmetril (Amantadine)

Tinkertoy Test

Tolerance

Vocational Counseling

Wisconsin Card Sorting Test

\section{Neuropsychological Evaluation-Jeff Kreutzer}

\section{Section Editor: Yana Suchy}

Agitated Behavior Scale

Behavioral Assessment of the Dysexecutive Syndrome

Boston Process Approach

Brief Cognitive Rating Scale

Cambridge Neuropsychological Testing Automated Battery 
Cognistat

Coma Recovery Scale

Coma/Near Coma Scale

Dot Counting Test

Double Simultaneous Stimulation

Dynamic Assessment

Finger Tapping Test

Finger to Nose Test

Fingertip Number-Writing Perception

Fixed Battery

Flexible Battery

Forced-Choice Test

Galveston Orientation and Amnesia Test

Go/No-Go Testing

Grooved Pegboard Test

Halstead Impairment Index

Halstead-Reitan Neuropsychological Test Battery

Hand Dynamometer

Hypothesis Testing Approach to Evaluation

Katz Adjustment Scale

Kaufman Assessment Battery for Children

Line Bisection

Luria Nebraska Neuropsychological Battery

Memory Assessment Scales

Mental Status Examination

Meyers Neuropsychological Battery

Mini-Mental State Exam

Neurobehavioral Assessment

Neurobehavioral Functioning Inventory

Neurobehavioral Rating Scale

Neuropsychological Assessment Battery

Neurosensory Center Comprehensive

Examination for Aphasia

Orientation Log

Portland Digit Recognition Test

Purdue Pegboard

Reitan-Indiana Aphasia Screening Test

Reitan-Klove Sensory Perceptual Examination

Repeatable Battery for the Assessment of

Neuropsychological Status

Rey 15 Item Test

Rivermead Behavioral Inattention Test

Seashore Rhythm Test

Serial Digit Learning

Serial Subtractions

Stroop Color Word Test (Adult)

Stroop Neuropsychological Screening Test (Adult)
Symptom Validity Assessment

Tactile Form Recognition

Tactual Performance Test

Test of Memory Malingering

Trail Making Test

Wide Range Achievement Test-4

Word Memory Test

Barrow Neuropsychological Screen

\section{Psychological Conditions-Bruce Caplan}

Section Editor: Robert G. Frank

Affect

Affective Disorder

Agitation

Alcohol Abuse

Alcohol Dependence

Alcoholic Brain Syndrome

Alcoholism

Alertness

Alexithymia

Anxiety

Behavior Management

Behavioral Assessment

Behavioral Therapy

Catastrophic Reaction

Circumstantiality

Clinical Practice Guidelines

Cogniform Disorder

Coping

De-escalation

Denial

Depressive Disorder

Distributed Systems

Emotional Lability

Family Adjustment

Family Burden

Flight of Ideas

Frustration Tolerance

Grandiosity

Hallucination

Hypersexuality/Hyposexuality

Ideas of Reference

Irritable Bowel Syndrome

La Belle Indifference

Major Depression

Masked Depression 
Medical Model

Mental Flexibility

Minor Depressive Disorder

Mood Disorder

Motivation

Narcissism

Neurotic Disorder

Neuroticism

Nonadherence

Noncompliance with Treatment

Pain

Paranoid Ideation

Paranoid Psychosis

Pica

Placebo

Positive Supports

Post-traumatic Stress Disorder

Premorbid Personality

Prescription Privilege

Pressured Speech

Psychogenic Disorder

Psychogenic Pain

Psychomotor Retardation

Psychosis

Psychotic Disorder

Redirection

Schizotypal Personality Disorder

Sex Therapy

Somatization

Stages of Adjustment

Stimulus Control

Stimulus Generalization

Stress

Substance Use Disorders

Suicidal Ideation

Tangentiality

Therapeutic Community

Therapist-Patient Relationship

Third Party Administrator

Double Dissociation

\section{Psychological Evaluation-Bruce Caplan}

Section Editor: Stephanie A. Reid-Arndt

Beck Anxiety Inventory

Beck Depression Inventory

Bell Curve

Brief Psychiatric Rating Scale
Brief Symptom Inventory

CAGE Questionnaire

Center for Epidemiological Studies: Depression

Clinical Interview

Clinical Significance

Computerized Assessment of Response Bias

Confounding Variables

Eysenck Personality Inventory

F Minus K Index

F Scale

Faking Good, Bad

Family Needs Questionnaire

Geriatric Depression Scale

Hamilton Depression Rating Scale

Hiscock Forced-Choice Test

House-Tree-Person Test

K Scale

L Scale

MacArthur Competence Assessment Tools

McGill Pain Questionnaire

McMaster Family Assessment Device

Michigan Alcoholism Screen Test

Millon Adolescent Clinical Inventory

Millon Behavioral Health Inventory

Millon Clinical Multiaxial Inventory

Minnesota Multiphasic Personality Inventory

Multiaxial Assessment

Myers-Briggs Inventories

NEO Personality Inventory

Patient Health Questionnaire

Personality Assessment Inventory

Projective Tests

Psychometrician

Rorschach

Satisfaction with Life Scale

Sentence Completion

Structured Clinical Interview for DSM-IV

(SCID-I/SCID-II)

Symptom Checklist-90-Revised

Thematic Apperception Test

True Response Inconsistency Scale (TRIN, MMPI)

Validity Scales (MMPI)

Variable Response Inconsistency Scale (VRIN, MMPI)

Victoria Symptom Validity Test

Vocational Assessment

Zung Self-Rating Depression Scale 
Positive and Negative Affect Schedule (PANAS)

Schedule for Nonadaptive and Adaptive

Personality (SNAP)

Alcohol Use Disorders Indentification Test

(AUDIT), The

King-Devick Test

Millon Behavioral Medicine Diagnostic

\section{Psychometrics-Jeff Kreutzer}

\section{Section Editor: Michael Franzen}

Age Decrements

Alternate Test Forms

Analysis of Variance

ANCOVA/MANCOVA

Barona Index

Base Rate (Population)

Battery Approach

Best Performance Method

Brain Reserve Capacity

Ceiling Effect

Chi-square

Classical Test Theory

Clustering

Confirmatory Bias

Contingency Table

Correlation Coefficients

Cultural Sensitivity

Culture Fair Test

Cutoff Scores, Cutting Scores

Deficit Measurement

Differential Diagnosis

Disability

Domain Referenced Test Interpretation

Dual Diagnosis

Ecological Validity

Error, Sources of

Factor Analysis

False Negative

False Positive

Floor Effect

Generalizability Theory

History (Medical, Social, Psychological)

Hold-Don't Hold Tests

Individual Comparison Standards

Inter-rater Reliability

Intertest Scatter
Intratest Scatter

Ipsative

Item Analysis

Item Difficulty

Item Response Theory

Kappa Coefficient

Kennard Principle

Latent Variable

Mayo's Older Americans Normative Studies (MOANS)

Measurement Theory

Meta-analysis

Modified Testing

Multiple Comparisons

Multiple Regression

Multivariate Analysis of Variance

Negative Predictive Power

Neuropsychological Deficit Scale

Neuropsychological Report

Neuropsychological Screening Examination

Nonparametric Statistics

Nonstandard Assessment

Normal Aging

Normal Curve

Normative Data

Observer Reports and Ratings

Parallel Forms

Path Analysis

Pattern Analysis

Percentiles

Phrenology

Positive Predictive Power

Premorbid Estimate

Premorbid Functioning

Qualified Examiner

Receiver-Operating Characteristics

Referral Question

Reliable Change Index

Response Bias

Self-Report Measures

Sensitivity

Specificity

Speed-Accuracy Tradeoff

Standard Error of the Mean

Standard Scores

Standardized Tests

Statistical Significance

Structural Equation Modeling 
Subtest Scatter

T Scores

Table of Specifications

Task Load

Test Bias

Test Construction

Test Interpretations: Computer Based

Test Reliability

Test Validity

Testing the Limits

Wonderlic Personnel Test

Z Scores

Basal and Ceiling Rules

\section{Psychopharmacology-John Deluca}

Section Editor: Efrain Antonio Gonzalez

Acetylcholine

Adrenocorticotropic Hormone

Alprazolam

Amitriptyline

Amoxapine

Amphetamine

Anticholinesterase Inhibitors

Antidepressants

Apolipoprotein E

Aripiprazole

Atomoxetine

Barbiturates

Benzodiazepines

Bromocriptine

Bupropion

Buspirone

Carbamazepine

Catecholamines

Chlordiazepoxide

Chlorpromazine

Citalopram

Clomipramine

Clonazepam

Clonidine

Clorazepate

Clozapine

Cmax

Compartmentalization

Contraindication

Cytochrome P450
D-Amphetamine

Desipramine

Dexamethasone

Dexmethylphenidate

Diazepam

Discontinuation Syndrome

Donepezil

Dopamine

Doxepin

Duloxetine

Electro-Convulsive Therapy

Enzyme

Epinephrine

Escitalopram

Extrapyramidal Symptoms

Fluoxetine

Fluphenazine

GABA

Gabapentin

Galantamine

Glutamate

Half-Life

Haloperidol

Hydroxyzine

Imipramine

Lamotrigine

Levetiracetam

Levodopa

Lithium

Lorazepam

L-Tryptophan

Maprotiline

Memantine

Mesoridazine

Methylphenidate

Midazolam

Mirtazapine

Moclobemide

Modafinil

Molindone

Monoamine Oxidase

Nefazodone

Neuropeptides

Neurotransmitters

N-Methyl-D-Aspartate (NMDA) Receptors

Norepinephrine

Nortriptyline

Olanzapine 
Oxazepam

Oxcarbazepine

Paroxetine

Pentobarbital

Perphenazine

$\mathrm{pH}$

Phenelzine

Phenytoin

Pimozide

Pregabalin

Quazepam

Quetiapine

Receptor Spectrum

Reserpine

Risperidone

Rivastigmine

Selective Serotonin Reuptake Inhibitors

Selegiline

Serotonin

Serotonin Syndrome

Sertraline

Tachyphylaxis

Tacrine

Temazepam

Thioridazine

Thiothixene

Tiagabine

Tmax

Topiramate

Tranylcypromine

Trazodone

Triazolam

Trifluoperazine

Trimipramine

Valproate

Venlafaxine

Zaleplon

Ziprasidone

Zolpidem

Zonisamide

Zopiclone

Deutetrabenazine

Asenapine

Brexipiprazole

Eszopiclone

Iloperidone

Fetzima (Levomilnacipran)

Tetrabenazine
Valbenazine

Vilazodone

Vortioxetine

Paliperidone

Pimavanserin

Lisdexamfetamine Dimesylate

Desvenlafaxine

Cariprazine

\section{Seizure-John Deluca}

Section Editor: Nancy D. Chiaravalloti

Absence Seizure

Aura

Complex Partial Seizure

Cortical Mapping

Epilepsy

Functional Magnetic Resonance Imaging

Grand Mal Seizure

Ictal Phenomena

Interictal Behavior Syndrome

Ischemic Penumbra

Kindling

Paroxysmal Disorder

Simple Partial Seizure

Petit Mal Seizure

Postictal Confusion

Psychomotor Epilepsy

Seizure

Status Epilepticus

Syncope

Temporal Lobe Epilepsy

\section{Sensory-Bruce Caplan}

Section Editor: John E. Mendoza and Bruce

Caplan

Accessory Cuneate Nucleus

Afferent Paresis

Ageusia

Ahylognosia

Allesthesia

Amorphognosis

Amusia

Anosodiaphoria

Anterolateral System

Apperceptive Visual Agnosia 
Asomatognosia

Association Areas

Associative Visual Agnosia

Auditory Agnosia

Auditory Cortex

Auditory System

Autotopagnosia

Body Schema

Brain Plasticity

Brown-Séquard Syndrome

Calcarine Fissure

Chief Sensory Nucleus of V

Cochlea

Cochlear Nuclei (Dorsal and Ventral)

Color Agnosia

Color Anomia

Color Imagery

Dejerine-Roussy Syndrome

Dermatome

Dorsal Nerve Roots

Dorsal Root Ganglia

Epicritic Pain

Equipotentiality

Fasciculus Cuneatus

Fasciculus Gracilis

Finger Agnosia

Functional Neuroanatomy

Gerstmann's Syndrome

Global Versus Local Processing

Graphesthesia

Heschl's Gyrus

Heteromodal Cortex

Homonymous Hemianopsia

Homonymous Quadrantanopsia

Homotypic Cortex

Hyperesthesia

Idiotypic Cortex

Inferior Colliculi

Kinesthesia

Lateral Lemniscus

Lingual Gyrus

Medial Geniculate Nuclei

Medial Lemniscus

Metamorphopsia

Meyer's Loop

Nucleus Cuneatus

Nucleus Gracilis

Nucleus Solitarius
Olfactory Bulb

Olfactory Tract

Optic Chiasm

Optic Nerve

Optic Radiations

Optic Tract

Pain Asymbolia

Palinopsia

Pallanesthesia

Pallesthesia

Paresis

Paresthesia

Peduncular Hallucinosis

Perception

Peripheral Neuropathy

Posterior Columns

Primary Cortex

Pronator Drift

Protopathic Pain

Referred Pain

Right Left Disorientation

Romberg Test

Secondary Cortex

Sensory Ataxia

Somatosensory Cortex

Somatotopic Organization

Somesthesia

Spatial Dyscalculia

Spinal Nucleus of V

Spinal Tract of V

Spinocerebellar Tracts

Spinoreticular Tract

Spinothalamic Tract

Substantia Gelatinosa

Superior Olivary Nucleus

Suppression

Synesthesia

Tactile Agnosia

Tactile Asymboly

Taste

Temperature, Perception of

Tertiary Cortex

Thalamic Pain Syndrome

Thermoanesthesia

Tinnitus

Topographical Disorientation

Touch, Localization of

Trapezoid Body 
Trigeminothalamic Tracts

Two-Point Discrimination

Unimodal Cortex

Ventral Posterior Lateral Nucleus

Ventral Posterior Medial Nucleus

Vertigo

Vestibular Dysfunction

Vestibular System

Vestibulocochlear Nerve

Visual Object Agnosia

Visual System

Weber Test

\section{Vascular-Bruce Caplan}

\section{Section Editor: Elliot Roth}

Aneurysm

Angioplasty

Anoxia

Anterior Cerebral Artery

Anterior Communicating Artery

Anticoagulation

Antiplatelet Therapy

Arteriovenous Malformation (AVM)

Atherosclerosis

Basilar Artery

Brainstem Strokes

Central Venous Thrombosis

Cerebellar Hemorrhage

Cerebral Amyloid Angiopathy

Cerebral Angiitis

Cerebral Blood Flow

Cerebral Embolism

Cerebrovascular Disease

Cholesterol

Circle of Willis

Collagen Vascular Disease

Congestive Heart Failure

Coronary Disease

Diaschisis

Diffusion-Weighted Imaging

Dissection

Echocardiogram

Hematoma

Hemorrhage

Hemorrhagic Stroke

Heparin

Hypertension
Infarction

Internal Carotid Artery

Intracerebral Hemorrhage

Intracranial Hemorrhage

Intraventricular Hemorrhage

Ischemia

Ischemic Stroke

L'etat Lacunaire

Lacunar Infarction

Lobar Hemorrhage

Lupus Cerebritis

Middle Cerebral Artery

Moyamoya Disease

Myocardial Infarction

NIH Stroke Scale

Perfusion-Weighted Imaging

Peripheral Vascular Disease

Posterior Cerebral Artery

Posterior Communicating Artery

Pure Motor Stroke

Recombinant Tissue Plasminogen Activator

Sickle-Cell Disease

Stent

Stroke

Subarachnoid Hemorrhage

Subdural Hematoma

Thalamic Hemorrhage

Thrombolysis

Thrombosis

Transcranial Doppler Ultrasonography

Transient Ischemic Attack

Vascular Malformations

Vasculitis

Vasospasm

Venous Thrombosis

Vertebrobasilar System

Warfarin (Coumadin)

Novel Oral Anticoagulants

Thrombectomy

Endovascular Therapy

Visual-John Deluca

Section Editor: Ronald Cohen and Adam J. Woods

Achromatopsia

Astereognosis

Balint's Syndrome 
Bicycle Drawing Test

Blindsight

Block Design

Calcarine Cortex

Capgras Syndrome

Charles Bonnet Syndrome

Clock Drawing

Closure

Conjugate Gaze

Cortical Blindness

Cortical Magnification

Cuneus

Diplopia

Dorsal Visual Pathway

Dorsomedial Visual Area

Edge Detection

Embedded Figures Test

Extrastriate

Facial Recognition Test

Feature Perception

Figure-Ground Discrimination

Fourier Transforms

Fovea

Gollin Figures

Hemianopia

Hemispatial Neglect

Hooper Visual Organization Test

Inferior Parietal Area

Judgment of Line Orientation

Lateral Geniculate Nucleus of the Thalamus

Lateral Inhibition

Macropsia

Magnocellular Neurons

Micropsia

Neglect Syndrome

Nystagmus

Object Recognition

Ocular Dominance

Oculomotor Nerve

Optic Ataxia

Optokinetic Reflex

Parvocellular Neurons

Prosopagnosia

Pseudobulbar Palsy

Pulvinar

Quadrantanopia

Receptive Fields

Retinopathy
Rey Complex Figure Test

Saccadic Eye Movements

Scotoma

Simultanagnosia

Spatial Frequency Analysis

Spatial Processing

Tachistoscopic Presentation

Temporal Inhibition

Trochlear Nerve

Ventral Visual Pathway

Visual Agnosia

Visual Angle

Visual Field Deficit

Visual Form Discrimination

Visual Hallucinations

Visual Modularity

Visual Neglect

Visual Tracking

Visual-Motor Function

Visual-Spatial Ability

Visuoperceptual

Macular Degeneration

Distance Perception

Binocular Disparity

Motion Parallax

Eye Dominance

Visual Convergence

Posterior Cortical Atrophy

Motion Perception

Visual Psychophysics

Just Noticeable Difference

Absolute Threshold

Appercetive Agnosia

Retinotopic Mapping

Spatial Sequence Synesthesia

Mach Bands

Backwards Masking

Post Trauma Vision Syndrome

Visual Assessment Battery (VAB)

Military Neuropsychology-Jeff Kreutzer

Section Editor: Risa Nakase-Richardson

Military Neuropsychology

Suicide

Emerging Consciousness Program

Military Deployment 
Types of Military Discharge

Military Occupational Status

Department of Defense (DoD)

Post-concussive Syndrome

Post-deployment Syndrome

Pre-deployment Cognitive Testing

Blast Effects

Decompression Sickness

Cognitive Resilience

Slick Criteria

Neurobehavioral Symptom Inventory

PCL-C

VA TBI Model System Program

Polytrauma System of Care

Agent Orange

Arterial Gas Embolism

Noncredible Symptom History

Jennifer Vasterling

Carrie Kennedy

Automated Neuropsychological Assessment

Metrics (ANAM)

Battlefield Assessment

Fitness-for-Duty or Physical Evaluation Board

Defense and Veterans Brain Injury Center

Polytrauma Transitional Rehabilitation Program

Chronic Effects of Neurotrauma Consortium

Vietnam Head Injury Study

Heat-Related Illness

Military Acute Concussion Evaluation

National Intrepid Center of Excellence

Louis French, Ph.D.

\section{Neuroimaging-John Deluca}

\section{Section Editor: Erin D. Bigler and Jo Ann}

Petrie

False Discovery Rate

Machine Learning

Shape/Countour Analysis

Quantitative Neuroimaging Analysis

Lesion-Symptom Mapping

Segmentation and Classification

Cortical Thickness

FreeSurfer

Lesion Burden

Surface Area

Susceptibility Weighted Imaging
Graph Theory

Network Connectivity

Network Edges

Network Hubs

Network Nodes

Rich Club Networks

Ventricle-to-Brain (VBR) Ratio

Voxel-Based Morphometry

Resting-State Functional Connectivity MRI (fcMRI)

Diffusion Kurtosis Mapping

PET Imaging

Near-Infrared Spectroscopy

Tract-Based Spatial Statistics (TBSS)

\section{DSM-5-Jeff Kreutzer}

\section{Section Editor: Ana Mills}

Social (Pragmatic) Communication Disorder Premenstrual Dysphoric Disorder

Disruptive Mood Dysregulation Disorder

Antidepressant Discontinuation Syndrome

Neurocognitive Disorders

OCD

Body Dysmorphic Disorder

Hoarding

Excoriation

Reactive Attachment Disorder

Acute Stress Disorder

Adjustment Disorder

Disinhibited Social Engagement Disorder

Illness Anxiety Disorder

Rumination Disorder

Anorexia Nervosa

Bulimia Nervosa

Avoidant/Restrictive Food Intake Disorder

Binge-Eating Disorder

Enuresis

Encopresis

Gender Dysphoria

Oppositional Defiant Disorder

Intermittent Explosive Disorder

Conduct Disorder

Pyromania

Kleptomania

Paranoid Personality Disorder

Schizoid Personality Disorder 
Antisocial Personality Disorder

Borderline Personality Disorder

Histrionic Personality Disorder

Narcissistic Personality Disorder

Dependent Personality Disorder

Paraphilic Disorders

Sexual Dysfunctions

Obsessive-Compulsive and Related Disorders

Trauma- and Stressor-Related Disorders

Somatic Symptom and Related Disorders
Feeding and Eating Disorders

Elimination Disorders

Disruptive, Impulse-Control, and Conduct

Disorders

Personality Disorders

Obsessive-Compulsive Personality Disorder

Somatic Symptom Disorder 


\section{About the Editors}

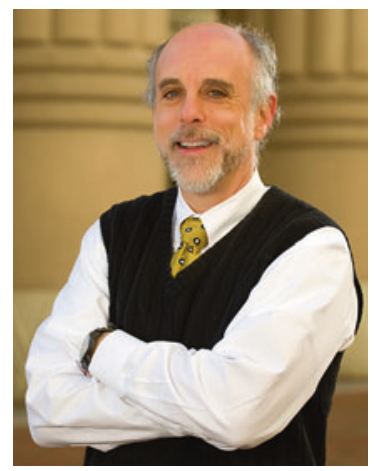

Jeffrey S. Kreutzer Department of Physical Medicine and Rehabilitation, Virginia Commonwealth University, Richmond, VA, USA

Dr. Jeffrey S. Kreutzer is the Rosa Schwarz Cifu Professor in the Department of Physical Medicine and Rehabilitation (PM\&R) at Virginia Commonwealth University's Medical College of Virginia Campus in Richmond. He first came to VCU as a postdoctoral fellow in 1982 after completing an internship in Neuropsychology and Family Therapy with Muriel Lezak at the Portland, Oregon VA Medical Center. He joined Virginia Commonwealth University's medical school faculty in the Fall of 1983.

Board certified in rehabilitation psychology (ABPP), Dr. Kreutzer has more than three decades of experience as a brain injury rehabilitation specialist. He was a founding member of the Virginia Head Injury Foundation, now known as BIAV. Since 1987, he has served as the Director of VCU's federally designated Traumatic Brain Injury Model System (TBIMS). As a clinician, Dr. Kreutzer provides neuropsychological and family support services to persons with a wide variety of neurological disabilities. His practice is holistic, emphasizing skills training, psychological support, and patient education.

Throughout his career, Dr. Kreutzer has been involved in developing empirically based intervention programs for persons with brain injury. For example, in 1986 he and Dr. Paul Wehman received a federal grant to adapt 
supported employment methods for persons with brain injury. Their successful program development along with very positive outcome data has helped make supported employment the current "standard of care" for TBI employment services. More recently, Dr. Kreutzer is developing empirically based programs to enhance resilience, couples' relationships, and family functioning.

Dr. Kreutzer has received numerous awards. In 1994, he received the Sheldon Berrol Clinical Service Award from The National Head Injury Foundation. In 2002, he received the Robert L. Moody Prize for Distinguished Initiatives in Brain Injury Research and Rehabilitation from the University of Texas. He has received two awards from the American Psychological Association, the Roger Barker Distinguished Research Contribution Award (2003) and the Diller Award for Demonstrated Excellence in the Field of Neurorehabilitation (2005). In 2010, he received the Distinguished Lifetime Contribution to Neuropsychology Award from the National Academy of Neuropsychology. In 2012, he received the McCollom Research Award from the Foundation for Life Care Planning Research, and in 2013 he received the Innovations in Treatment Award from the North American Brain Injury Society. In 2017, he was presented with the Jennett Plum Award for Clinical Achievement in the Field of Brain Injury Medicine by the International Brain Injury Association.

A frequent speaker at national and international meetings, he has given more than 500 invited presentations. A Fellow in the American Congress of Rehabilitation Medicine (FACRM), National Academy of Neuropsychology, and the American Psychological Association, Dr. Kreutzer has co-authored more than 170 publications, mostly in the area of traumatic brain injury and rehabilitation. Editor-in-Chief of two international journals, Brain Injury and NeuroRehabilitation, he has also published more than 15 books covering topics such as family intervention, vocational rehabilitation, community reintegration, behavior management, and cognitive rehabilitation.

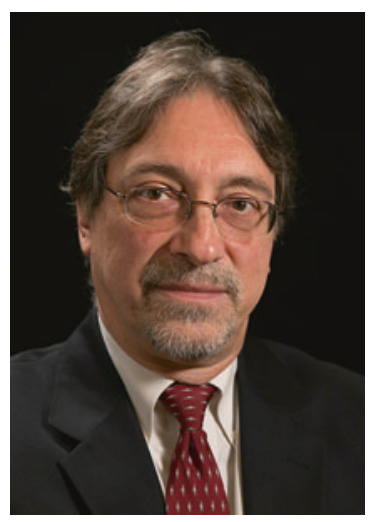

John DeLuca is the Senior Vice President for Research and Training at Kessler Foundation, a Professor in the Department of Physical Medicine and Rehabilitation, and the Department of Neurology at Rutgers, New Jersey 
Medical School. He is a licensed Psychologist in New Jersey and New York and is board certified in Rehabilitation Psychology by the American Board of Professional Psychology. Dr. DeLuca has been involved in neuropsychology and rehabilitation research for over 25 years. He is internationally known for his research on disorders of memory and information processing in a variety of clinical populations, including multiple sclerosis, traumatic brain injury, aneurysmal subarachnoid hemorrhage, and chronic fatigue syndrome. Dr. DeLuca has published over 300 articles and chapters in these areas; has edited 5 books in neuropsychology, neuroimaging, and rehabilitation; and is a co-editor for the Encyclopedia of Clinical Neuropsychology. He has received over 32 million dollars in grant support for his research. Dr. DeLuca's most recent research ventures include the cerebral mapping of human cognitive processes using functional neuroimaging, as well as the development of research-based techniques to improve cognitive impairment. He serves as an Associate Editor of several journals and is on the editorial boards of many other journals. He is the recipient of several awards in recognition of his work including the 2015 Arthur Benton Award from the International Neuropsychological Society and 2012 Rodger G. Barker Distinguished Research Contribution Award from Division 22 (Rehabilitation Psychology) of the American Psychological Association. Dr. DeLuca has been very involved for many years in the training of postdoctoral fellows in neuropsychology and rehabilitation and has directed several advanced research and training programs sponsored by NIDRR, the National MS Society, and NIH since 1990.

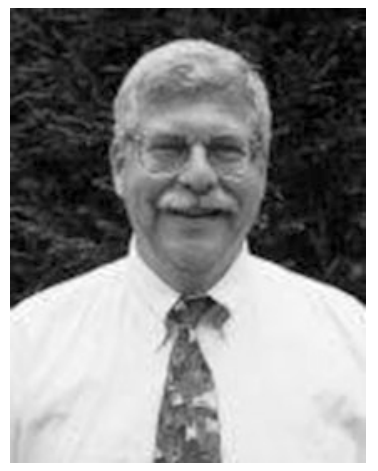

Bruce Caplan is board certified in clinical neuropsychology and rehabilitation psychology by the American Board of Professional Psychology and is a Fellow of the American Psychological Association (APA), National Academy of Neuropsychology, and American Congress of Rehabilitation Medicine. Dr. Caplan serves as Senior Editor of Journal of Head Trauma Rehabilitation, is a member of the Editorial Board of Topics in Stroke Rehabilitation, and previously served as Editor of Rehabilitation Psychology. In 1987, Dr. Caplan published Rehabilitation Psychology Desk Reference, the first edited textbook in the specialty. He is past President of the Philadelphia Neuropsychology Society and of Division 22 (Rehabilitation Psychology) of the APA. He is the recipient of two Distinguished Service Awards and the Lifetime Achievement 
Award from Division 22. Dr. Caplan was a founding member of the American Board of Rehabilitation Psychology. Currently in independent practice, he was formerly Professor and Chief Psychologist in the Department of Rehabilitation Medicine at Jefferson Medical College in Philadelphia. 


\section{Contributors}

Galya Abdrakhmanova University of Richmond, Richmond, VA, USA

Thomas M. Achenbach Department of Psychiatry, University of Vermont, Burlington, VT, USA

Russell Adams Department of Psychiatry and Behavioral Science, University of Oklahoma Health Sciences Center, Oklahoma City, OK, USA

Adrienne Adler Department of Neurobiology and Anatomy, Wake Forest School of Medicine, Winston-Salem, NC, USA

Adrienne Adler-Neal Department of Neurobiology and Anatomy, Wake Forest School of Medicine, Winston-Salem, NC, USA

Amma A. Agyemang Department of Physical Medicine and Rehabilitation, Virginia Commonwealth University Medical Center, Richmond, VA, USA

Cristy Akins Mercy Family Center, Metarie, LA, USA

Amy Alderson Department of Rehabilitation Medicine, Emory University, Atlanta, GA, USA

Brittany J. Allen Cox Health System, Springfield, MO, USA

Daniel N. Allen Department of Psychology, University of Nevada, Las Vegas, NV, USA

H. Allison Bender Department of Neurology, Icahn School of Medicine at Mount Sinai, New York, NY, USA

Ahmad Alsemari Psychology, Edgewood College, Madison, WI, USA

Karin Alterescu Department of Psychology, Queens College of the City University of New York (CUNY), Flushing, NY, USA

Robert A. Altmann A-PsychEd Publication Services, Minneapolis, MN, USA

Akshay Amaraneni Department of Pharmacology, Virginia Commonwealth University, Richmond, VA, USA 
Melissa Amick Department of Psychiatry and Human Behavior, Brown University, Providence, RI, USA

Aaron Andersen Utah State University, Logan, UT, USA

Heather Anderson Department of Neurology, University of Kansas School of Medicine, Kansas City, KS, USA

Steven W. Anderson University of Iowa Hospitals and Clinics, Iowa City, Iowa, USA

Kevin M. Antshel Department of Psychology, Syracuse University, Syracuse, NY, USA

Jennifer Ann Niskala Apps Department of Psychiatry and Behavioral Medicine, Children's Hospital of Wisconsin/Medical College of Wisconsin, Milwaukee, WI, USA

Juan Carlos Arango-Lasprilla IKERBASQUE, Basque Foundation for Science, Biocruces Research Institute, Bilbao, Spain

Amy J. Armstrong Department of Rehabilitation Counseling, Virginia Commonwealth University, Richmond, VA, USA

Carol L. Armstrong Child and Adolescent Psychiatry and Behavioral Sciences, The Children's Hospital of Philadelphia, Philadelphia, PA, USA

Glenn S. Ashkanazi Department of Clinical and Health Psychology, Clinical and Health Psychology Clinic, College of Public Health and Health Professions, University of Florida, Gainesville, FL, USA

Jane Austin Department of Psychology, William Paterson University, Wayne, NJ, USA

Bradley N. Axelrod John D. Dingell VA Medical Center, Psychology Section (11MHPS), Detroit, MI, USA

Lindsay E. Ayearst Multi-Health Systems Inc., Toronto, ON, Canada

Glen P. Aylward SIU School of Medicine- Developmental-Behavioral Pediatrics, Springfield, IL, USA

Samantha Backhaus Department of Neuropsychology, Rehabilitation Hospital of Indiana, Indianapolis, IN, USA

Erin Bailey Department of Psychology, Miami VA Healthcare System, Miami, FL, USA

Maya Balamane Mount Sinai Brain Injury Research Center, San Francisco, CA, USA

Angel Ball Department of Clinical Health Sciences, Texas A\&M University Kingsville, Kingsville, TX, USA

Sandra Banks Department of Psychiatry, Allegheny General Hospital, Pittsburgh, PA, USA 
James H. Baños Department of Medical Education, University of Alabama School of Medicine, Birmingham, AL, USA

Russell A. Barkley Virginia Treatment Center for Children and Virginia Commonwealth University Medical Center, Richmond, VA, USA

Ida Sue Baron Potomac, MD, USA

Erika M. Baron Pace University, New York, NY, USA

Mark S. Baron Department of Neurology, Virginia Commonwealth University Parkinson's and Movement Disorders Center, Richmond, Virginia, USA

Walter Barr Department of Psychology, William Paterson University, Wayne, NJ, USA

William B. Barr NYU Langone Medical Center, Comprehensive Epilepsy Center, New York, NY, USA

Joseph Barrash Department of Neurology, University of Iowa, Iowa City, IA, USA

Russell M. Bauer Department of Clinical and Health Psychology, University of Florida, Gainesville, FL, USA

Allison Baylor Virginia Commonwealth University, Richmond, VA, USA

Jessica Bean Department of Psychology, University of Connecticut, Storrs, CT, USA

Megan Becker Department of Psychology, University of Nevada, Las Vegas, NV, USA

James T. Becker Department of Psychiatry, University of Pittsburgh School of Medicine, WPIC, Pittsburgh, PA, USA

Pélagie M. Beeson Department of Speech, Language, and Hearing Sciences, The University of Arizona, Tucson, AZ, USA

Jay Behel Department of Behavioral Sciences, Rush University Medical Center, Chicago, IL, USA

Stephanie Behrens Department of Psychology, Utah State University, Logan, UT, USA

Stacy Belkonen Salem VA Medical Center, Salem, VA, USA

Department of Psychiatry and Behavioral Medicine, Virginia Tech Carilion School of Medicine, Roanoke, VA, USA

Andrew Bell Department of Anatomy and Neurobiology, Virginia Commonwealth University, Richmond, VA, USA

Brian D. Bell Department of Neurology, University of Wisconsin, Madison, WI, USA

Heidi A. Bender Department of Neurology, Icahn School of Medicine at Mount Sinai, New York, NY, USA 
Daniel B. Berch Curry School of Education, University of Virginia, Charlottesville, VA, USA

Brianne Magouirk Bettcher Department of Neurosurgery and Neurology, University of Colorado School of Medicine, Denver, CO, USA

Rocky Mountain Alzheimer's Disease Center, Aurora, CO, USA

Alyssa Beukema School Psychology, The Chicago School of Professional Psychology, Chicago, IL, USA

John Bigbee Anatomy and Neurobiology, Virginia Commonwealth University, Richmond, VA, USA

Erin D. Bigler Department of Psychology and the Neuroscience Center, Brigham Young University, Provo, UT, USA

Rebecca Bind Department of Neurology, Mount Sinai Medical Center, New York, NY, USA

J. Audie Black Allegheny General Hospital, Pittsburgh, PA, USA

Department of Psychiatry; Neuropsychology Section, Allegheny Health Network, Pittsburgh, PA, USA

Margaret Lehman Blake Communication Sciences and Disorders, University of Houston, Houston, TX, USA

Michelle L. Block Stark Neuroscience Research Institute, Indiana University, Indianapolis, IN, USA

Henrike K. Blumenfeld School of Speech, Language and Hearing Sciences, San Diego State University, San Diego, CA, USA

Ty Bodily Psychology Department and the Neuroscience Center, Brigham Young University, Provo, UT, USA

Doug Bodin Department of Pediatrics, Nationwide Children's Hospital and The Ohio State University, Columbus, OH, USA

Angela M. Bodling Center for Health Care Quality, University of MissouriColumbia, Columbia, MO, USA

Jennifer Bogner Department of Physical Medicine and Rehabilitation, Ohio State University, Columbus, OH, USA

Robert J. Boland Department of Psychiatry, Brigham and Women's Hospital/Harvard Medical School, Boston, MA, USA

Mark W. Bondi University of California San Diego School of Medicine, La Jolla, CA, USA

Neuropsychological Assessment Unit, VA San Diego Healthcare System, San Diego, CA, USA

Amanda D. Bono Department of Psychology, Queens College and The Graduate Center of the City University of New York, Flushing, NY, USA 
John G. Borkowski Department of Psychology, University of Notre Dame, Notre Dame, IN, USA

Joan C. Borod Department of Psychology, Queens College and The Graduate Center of the City University of New York (CUNY), New York, NY, USA Department of Neurology, Icahn School of Medicine at Mount Sinai, New York, NY, USA

Beth Borosh Cognitive/Behavioral Neurology Center Northwestern Feinburg School of Medicine, Chicago, IL, USA

Carley Borza University of Alberta, Edmonton, AB, Canada

Dawn E. Bouman Neuropsychology and Medical Psychology, University of Cincinnati, Department of Neurology and Rehabilitation Medicine, Cincinnati, $\mathrm{OH}, \mathrm{USA}$

Isabelle Bourdeau Division of Endocrinology, Department of Medicine, Research Centre, Centre hospitalier de l'université de Montréal (CHUM), Montreal, QC, Canada

Jessica Bove Emory University, Atlanta, GA, USA

Dawn Bowers Department of Clinical and Health Psychology, University of Florida, Gainesville, FL, USA

Mary Boyle The College of Humanities and Social Sciences, Montclair State University, Montclair, NJ, USA

Lauren E. Bradley Department of Behavioral Sciences, Rush University Medical Center, Chicago, IL, USA

Natalie Brei Department of Psychology, University of Wisconsin-Milwaukee, Milwaukee, WI, USA

Lisa A. Brenner Rocky Mountain Mental Illness Research Education and Clinical Center, Denver, CO, USA

University of Colorado, Anschutz Medical Campus, Aurora, CO, USA

Rachel M. Bridges Department of Psychology, University of South Carolina, Columbia, SC, USA

Andrew Brodbelt The Walton Centre NHS Foundation Trust, Liverpool, UK

Justine S. Broecker The Westminster Schools, Atlanta, GA, USA

Alyssa M. Broomfield School of Psychology, The Chicago School of Professional Psychology, Chicago, IL, USA

John Brown Medical College of Georgia, Augusta, GA, USA

Margaret Brown Mount Sinai School of Medicine, New York, NY, USA

Racine Brown Center of Innovation on Disability and Rehabilitation Research, James A. Haley Veterans' Hospital, Tampa, FL, USA 
Martha Brownlee-Duffeck Harry S. Truman Memorial, Columbia, MO, USA

Kelly Broxterman School Psychology, The Chicago School of Professional Psychology, Chicago, IL, USA

Hugh W. Buckingham Department of Communication Sciences and Disorders, College of Allied Health Sciences, University of Oklahoma Health Sciences Center, Oklahoma City, OK, USA

Ashley Bujalski Department of Psychology, William Paterson University, Wayne, NJ, USA

Rebecca Burger-Caplan Yale Child Study Center, Yale University School of Medicine, New Haven, CT, USA

Department of Pediatrics, Emory University School of Medicine, Atlanta, GA, USA

Jeffrey M. Burns Department of Neurology, University of Kansas School of Medicine, Kansas City, KS, USA

Thomas G. Burns Department of Neuropsychology, Children's Healthcare of Atlanta, Atlanta, GA, USA

Shane S. Bush Long Island Neuropsychology, P.C., Lake Ronkonkoma, NY, USA

Tamara Bushnik Inter-Hospital Research and Knowledge Translation, Rusk Rehabilitation, New York, NY, USA

Melissa Buttaro Department of Psychiatry, The Miriam Hospital, Providence, RI, USA

Meryl A. Butters Department of Psychiatry, University of Pittsburgh School of Medicine, WPIC, Pittsburgh, PA, USA

Xavier E. Cagigas UCLA Semel Institute for Neuroscience and Human Behavior, Los Angeles, CA, USA

Medical Psychology Assessment Center, Resnick Neuropsychiatric Hospital at UCLA, Los Angeles, CA, USA

Department of Psychiatry and Biobehavioral Sciences, David Geffen School of Medicine at UCLA, Los Angeles, CA, USA

Deborah A. Cahn-Weiner Department of Neurology, University of California, Davis, Davis, CA, USA

Rebecca M. Cain Fairfax Neonatal Associates, Fredericksburg, VA, USA

Charles D. Callahan Memorial Health System, Springfield, IL, USA

Jonathan Campbell Neurology, Virginia Commonwealth University, Richmond, VA, USA

Bruce Caplan Independent Practice, Wynnewood, PA, USA 
Jessica A. Carboni Counseling and Psychological Services, Georgia State University, Atlanta, GA, USA

Noelle E. Carlozzi Physical Medicine and Rehabilitation, University of Michigan, Ann Arbor, MI, USA

Helen M. Carmine ReMed, Paoli, PA, USA

Dominic A. Carone University Hospital - Neuropsychology Assessment Program, SUNY Upstate Medical University, Syracuse, NY, USA

Janessa O. Carvalho Department of Psychology, Bridgewater State University, Bridgewater, MA, USA

Jennifer Cass Department of Pediatrics, Nationwide Children's Hospital and The Ohio State University, Columbus, OH, USA

Adam Cassidy Harvard University, Cambridge, MA, USA

Eric Catlin Physical Medicine and Rehabilitation, University of South Florida, Tampa, FL, USA

Amiram Catz Department of Spinal Rehabilitation, Loewenstein Rehabilitation Hospital, Raanana, Israel

Tel-Aviv University, Tel-Aviv, Israel

Jessica Chaiken HeiTech Services, Inc., National Rehabilitation Information Center, Landover, MD, USA

Grace-Anna Chaney Department of Psychology, Towson University, Towson, MD, USA

Lanshin Chang University of Macau, Taipa, Macau SAR, China

Sandra Bond Chapman Center for BrainHealth, The University of Texas at Dallas' school of Behavioral and Brain Sciences, Dallas, TX, USA

Jesse Chasman Department of Psychology, University of Connecticut, Storrs, CT, USA

Sandy Sut Ieng Cheang Department of Psychology, University of Macau, Taipa, Macau, SAR, China

Colby Chlebowski Department of Psychiatry, University of California, San Diego, USA

Woon N. Chow Department of Pathology, Microbiology, and Immunology, Vanderbilt University Medical Center, Nashville, TN, USA

Shawn E. Christ University of Missouri, Columbia, MO, USA

Sarah S. Christman Buckingham Department of Communication Sciences and Disorders, The University of Oklahoma Health Sciences Center, Oklahoma City, OK, USA

Severn B. Churn Neurology, Virginia Commonwealth University, Richmond, VA, USA 
Angela Hein Ciccia Department of Psychological Sciences, Program in Communication Sciences, Case Western Reserve University, Cleveland, $\mathrm{OH}$, USA

Elaine Clark Department of Educational Psychology, The University of Utah, Salt Lake City, UT, USA

Lee Anna Clark Department of Psychology, University of Notre Dame, Notre Dame, IN, USA

Robert Clark School Psychology, The Chicago School of Professional Psychology, Chicago, IL, USA

Uraina S. Clark Department of Neurology, Icahn School of Medicine at Mount Sinai, New York, NY, USA

Dave Clarke Comprehensive Epilepsy Program, Dell Children's Medical Center of Central Texas, Austin, TX, USA

Department of Pediatrics, Dell Medical School, The University of Texas at Austin, Austin, TX, USA

Lauren Clevenger University of Iowa, Iowa City, IA, USA

Derin Cobia Department of Psychology and Neuroscience Center, Brigham Young University, Provo, UT, USA

Melanie M. Cochrane Department of Psychology, University of Victoria, Victoria, BC, Canada

Ronald A. Cohen Department of Clinical and Health Psychology, College of Public Health and Health Professions, University of Florida, Gainesville, FL, USA

Center for Cognitive Aging and Memory, McKnight Brain Institute, University of Florida, Gainesville, FL, USA

Morris J. Cohen Neurology, Pediatrics and Psychiatry, Pediatric Neuropsychology, Medical College of Georgia and BT-2601 Children's Medical Center, Augusta, GA, USA

Raymond J. Colello Anatomy and Neurobiology, Virginia Commonwealth University, Richmond, VA, USA

G. Combs Legal Psychology (Psychology and Law), Neuropsychology, Clinical Psychology, Chicago School of Professional Psychology, Chicago, IL, USA

Brian Comly Magee Rehabilitation Hospital, Philadelphia, PA, USA

Adam Conley Virginia Commonwealth University Medical Center, Richmond, VA, USA

Lori G. Cook Center for BrainHealth, The University of Texas at Dallas, Dallas, TX, USA 
Andrea Coppens Division of Neurology and Department of Psychology, Sick Kids-Centre for Brain and Mental Health, Toronto, ON, Canada

Patrick Coppens Communication Sciences and Disorders, SUNY Plattsburgh, Plattsburgh, NY, USA

Melinda A. Cornwell Department of Psychology, Queens College and The Graduate Center of the City University of New York, Flushing, NY, USA

Stephen Correia Department of Psychiatry and Human Behavior, Alpert Medical School, Brown University, Providence, USA

John D. Corrigan Department of Physical Medicine and Rehabilitation, Ohio State University, Columbus, OH, USA

Joyce A. Corsica Department of Behavioral Sciences, Rush University Medical Center, Chicago, IL, USA

H. Branch Coslett Department of Neurology, University of Pennsylvania, HUP, Philadelphia, PA, USA

Kelly Coulehan Health Psychology, Neuropsychology, Clinical Psychology, Fordham University, New York, NY, USA

John C. Courtney Socorro Mental Health, Presbyterian Medical Services, Socorro, NM, USA

David R. Cox Neuropsychology and Rehabilitation Consultants, P.C., Chapel Hill, NC, USA

Laura Cramer-Berness Department of Psychology, William Paterson University, Wayne, NJ, USA

Chava Creque Department of Psychology and Neuroscience, University of Colorado Boulder, Boulder, CO, USA

Savannah Crippen Department of Psychology, William Paterson University, Wayne, NJ, USA

Jessica Cruz School Psychology, The Chicago School of Professional Psychology, Chicago, IL, USA

Yenisel Cruz-Almeida Pain Research and Intervention Center of Excellence Clinical and Translational Science Institute, University of Florida, Gainesville, FL, USA

Carter M. Cunningham Fairfax Neonatal Associates, Falls Church, VA, USA

Jacqueline L. Cunningham Department of Psychology, Children's Hospital of Philadelphia, Philadelphia, PA, USA

Sean Cunningham Department of Educational Psychology, University of Utah, Salt Lake City, UT, USA

Cody Curatolo Department of Psychology, William Paterson University, Wayne, NJ, USA 
Cherina Cyborski National Intrepid Center of Excellence, Walter Reed National Military Medical Center, Bethesda, MD, USA

Rik Carl D'Amato School Psychology, Clinical Neuropsychology, Clinical Psychology, The Chicago School of Professional Psychology, Chicago, IL, USA

Kristen Dams-O'Connor Department of Rehabilitation Medicine, Mount Sinai School of Medicine, New York, NY, USA

Natalie Dattilo Department of Psychiatry, Indiana University School of Medicine, Indianapolis, IN, USA

Andrew S. Davis Department of Educational Psychology, Ball State University, Muncie, IN, USA

Marcus Ponce de Leon Madigan Army Medical Center, Tacoma, WA, USA

Ashley de Marchena Department of Psychology, University of Connecticut, Storrs, CT, USA

Scott L. Decker Department of Psychology, University of South Carolina, Columbia, SC, USA

Nathalie DeFabrique Cook County Department of Corrections, Chicago, IL, USA

Nick A. DeFilippis Georgia School of Professional Psychology, Atlanta Psychological Associates, Atlanta, GA, USA

Kathleen K. M. Deidrick Neuro- and Behavioral Psychology, St. Luke's Children's Hospital, Boise, ID, USA

Lisa Delano-Wood Memory, Aging and Resilience Clinic (MARC), Department of Psychiatry, University of California, San Diego, La Jolla, CA, USA

VA San Diego Healthcare System, San Diego, CA, USA

Dean C. Delis School of Medicine, University of California-San Diego, La Jolla, CA, USA

John DeLuca Research Department, Kessler Foundation, West Orange, NJ, USA

George J. Demakis Department of Psychology, University of North Carolina at Charlotte, Charlotte, NC, USA

Theslee Joy DePiero Braintree Rehabilitation Hospital, Boston University School of Medicine, Boston, MA, USA

Roberta DePompei School of Speech-Language Pathology and Audiology, University of Akron, Akron, OH, USA

Emily Desbiens Department of Psychology, William Paterson University, Wayne, NJ, USA 
Krista Dettle Department of Psychology, William Paterson University, Wayne, NJ, USA

Bruce J. Diamond Department of Psychology, William Paterson University, Wayne, NJ, USA

Aimee Dietz Communication Sciences and Disorders, University of Cincinnati, Cincinnati, $\mathrm{OH}$, USA

María Díez-Cirarda Department of Methods and Experimental Psychology, Faculty of Psychology and Education, University of Deusto, Bilbao, Spain

Marcel P. J. M. Dijkers American Congress of Rehabilitation Medicine, Reston, VA, USA

Department of Rehabilitation Medicine, Icahn School of Medicine at Mount Sinai, New York, NY, USA

Ekaterina Dobryakova Traumatic Brain Injury Research, Kessler Foundation, West Orange, NJ, USA

Carl B. Dodrill Department of Neurology, University of Washington School of Medicine, Seattle, WA, USA

Ben Dodsworth PM\&R, University of South Florida, Tampa, FL, USA

Peter Dodzik Fort Wayne Neurological Center, Fort Wayne, IN, USA

Christopher H. Domen School of Medicine, Department of Neurosurgery, University of Colorado, Aurora, CO, USA

Jacobus Donders Mary Free Bed Rehabilitation Hospital, Grand Rapids, MI, USA

Kerry Donnelly VA WNY Healthcare System, University of Buffalo (SUNY) Behavioral Health Careline (116B), Buffalo, NY, USA

Jennifer M. Doran VA Connecticut Healthcare System, Yale School of Medicine, Newington, CT, USA

Vonetta Dotson Department of Psychology, Georgia State University, Atlanta, GA, USA

Department of Clinical and Health Psychology College of Public Health and Health Professions, University of Florida, Gainesville, FL, USA

Lauren R. Dowell Laboratory for Neurocognitive and Imaging Research, Kennedy Krieger Institute, Baltimore, MD, USA

Leah Drasher-Phillips Center of Innovation on Disability and Rehabilitation Research, James A. Haley Veterans' Hospital, Tampa, FL, USA

Elena Harlan Drewel Neuro- and Behavioral Psychology, St. Luke's Children's Hospital, Boise, ID, USA 
Tina Drossos Departments of Psychiatry and Behavioral Neuroscience, and Pediatrics, Section of Child and Adolescent Psychiatry, The University of Chicago, Chicago, IL, USA

Lindsey Duca Department of Orthopedics and Rehabilitation, University of Wisconsin School of Medicine and Public Health, Madison, WI, USA

Nicole M. Dudukovic Department of Psychology, University of Oregon, Eugene, OR, USA

Mary Dunkle National Organization for Rare Disorders (NORD), Danbury, CT, USA

Kari Dunning Department of Rehabilitation Sciences, University of Cincinnati, Cincinnati, OH, USA

Jeff Dupree Anatomy and Neurobiology, Virginia Commonwealth University, Richmond, VA, USA

Ana Durand Sanchez Physical Medicine and Rehabilitation, Baylor College of Medicine, Houston, TX, USA

Moira C. Dux US Department of Veteran Affairs, Baltimore, MD, USA

Blessen C. Eapen Polytrauma Rehabilitation Center, South Texas Veterans Health Care System, San Antonio, TX, USA

Department of Rehabilitation Medicine, UT Health San Antonio, San Antonio, TX, USA

Angela Eastvold Department of Neurology, The University of Utah, Salt Lake City, UT, USA

Natalie C. Ebner Department of Psychology, University of Florida, Gainesville, FL, USA

Department of Aging and Geriatric Research, Institute on Aging, University of Florida, Gainesville, FL, USA

Lisa Edmonds Communication Sciences and Disorders, Teachers College Columbia University, New York, NY, USA

Dawn M. Ehde Department of Rehabilitation Medicine, University of Washington, Seattle, WA, USA

Alyssa Eidson Emory University/Rehabilitation Medicine, Atlanta, GA, USA

Donovan Ellis Department of Psychology, University of Florida, Gainesville, FL, USA

Erin E. Emery-Tiburcio Department of Behavioral Sciences, Rush University Medical Center, Chicago, IL, USA

Joel Eppig Joint Doctoral Program in Clinical Psychology, University of California, San Diego State University, San Diego, CA, USA 
Jacqueline Estrada School of Psychology, The Chicago School of Professional Psychology, Chicago, IL, USA

Eleazar Eusebio School Psychology, The Chicago School of Professional Psychology, Chicago, IL, USA

Allison S. Evans Concord Comprehensive Neuropsychological Services, Concord, MA, USA

Daniel Erik Everhart Department of Psychology, Eastern Carolina University, Greenville, NC, USA

Nathan Ewigman Mental Health Service Line, San Francisco VA Health Care System, San Francisco, CA, USA

Joseph E. Fair Brigham Young University, Provo, UT, USA

Jacob Faltin School Psychology, The Chicago School of Professional Psychology, Chicago, IL, USA

Jaelyn R. Farris Department of Psychology, Youngstown State University, Youngstown, OH, USA

Khan Fary Department of Medicine, University of Melbourne and the Royal Melbourne Hospital, Parkville, VA, Australia

Deborah Fein Department of Psychological Sciences, University of Connecticut, Storrs, CT, USA

Leilani Feliciano Department of Psychology, University of Colorado at Colorado Springs, Colorado Springs, CO, USA

Aaron Feliu James A Haley Veterans Hospital, Tampa, FL, USA

Warren L. Felton Department of Neurology, Virginia Commonwealth University Medical Center, Richmond, VA, USA

Kyle E. Ferguson University of British Columbia, Vancouver, BC, Canada

Robert Fieo Department of Aging and Geriatric Research, College of Medicine, Institute on Aging, University of Florida, Gainesville, FL, USA

Christina Figueroa Memory and Aging Program, Butler Hospital, Providence, RI, USA

Carole M. Filangieri Department of Behavioral Health, NYU Winthrop Hospital, Mineola, NY, USA

Eric M. Fine Department of Neurology, University of California, San Francisco, CA, USA

Jessica Fish Medical Research Council Cognition and Brain Sciences Unit, Cambridge, UK

Julie Testa Flaada Rochester, MN, USA

Jennifer Fleming School of Health and Rehabilitation Sciences, The University of Queensland, Brisbane, QLD, Australia 
Kimberly Fleming Department of Psychiatry and Behavioral Sciences, The University of Kansas KU Medical Center, Kansas City, KS, USA

James R. Flynn Department of Politics, The University of Otago, Dunedin, New Zealand

Kristin Joan Flynn Peters Department of Health Sciences, University of Missouri, Columbia, MO, USA

Nancy S. Foldi Department of Psychology, Queens College and The Graduate Center, The City University of New York, New York, NY, USA

Department of Medicine, Winthrop University Hospital, Stony Brook School of Medicine, Mineola, NY, USA

Samantha Foreman Immaculata University, Immaculata, PA, USA

Hélène Forget Département de psychoéducation et de psychologie, Université du Québec en Outaouais, Gatineau, QC, Canada

Bonny J. Forrest Independent Practice, San Diego, CA, USA

Lisa M. Fox NYU Langone Medical Center, Department of Psychology, Rusk Institute of Rehabilitation Medicine, New York, NY, USA

Michael A. Fox Anatomy and Neurobiology, Virginia Commonwealth University Medical Center, Richmond, VA, USA

Laura L. Frakey Memorial Hospital of Rhode Island and Alpert Medical School of Brown University, Pawtucket, RI, USA

Robert G. Frank University of New Mexico, Albuquerque, NM, USA

Michael Franzen Allegheny General Hospital, Pittsburgh, PA, USA

Stacy Frauwirth Academic Therapy Publications, Novato, CA, USA

Ian Frazier Department of Psychology, University of Florida, Gainesville, FL, USA

Kathleen L. Fuchs Department of Neurology, University of Virginia Health System, Charlottesville, VA, USA

Shawn Gale Department of Psychology and Neuroscience Center, Brigham Young University, Provo, UT, USA

Kristin Galetta Department of Neurology, Brigham and Women's Hospital, Boston, MA, USA

Steven Galetta Department of Neurology, New York University of Medicine, New York, NY, USA

Sherri Gallagher Wellness PSI, LLC Private Practice, Flagstaff, AZ, USA

Frank J. Gallo Department of Psychology, University of WisconsinMilwaukee, Milwaukee, WI, USA 
Emnet Gammada Department of Psychology, Queens College and The Graduate Center, The City University of New York, New York, NY, USA

Sarah Garcia Department of Psychiatry- Neuropsychology, University of Michigan Health System, Ann Arbor, MI, USA

Pamela Garn-Nunn Department of Pharmacology, Virginia Commonwealth University, Richmond, VA, USA

Kelly Davis Garrett Intermountain Healthcare and University of Utah Center on Aging, Salt Lake City, UT, USA

Kelli Williams Gary Department of Occupational Therapy, Virginia Commonwealth University, Richmond, VA, USA

Brandon E. Gavett Department of Psychology, University of Colorado Colorado Springs, Colorado Springs, CO, USA

Helen M. Genova Neuropsychology and Neuroscience Laboratory, Kessler Foundation Research Center, West Orange, NJ, USA

Savannah J. Geske Department of Psychiatry and Behavioral Sciences, University of Kansas Medical Center, Kansas City, KS, USA

Glen E. Getz Department of Psychiatry, Allegheny General Hospital, Pittsburgh, PA, USA

Neuropsychology Specialty Care, LLC, Pittsburgh, PA, USA

Christine Ghilain Department of Neuropsychology, Children's Healthcare of Atlanta, Atlanta, Georgia, USA

Gerard A. Gioia Children's National Medical Center, Rockville, MD, USA

Elizabeth Louise Glisky Department of Psychology, University of Arizona, Tucson, AZ, USA

Emilie Godwin Virginia Commonwealth University, Richmond, VA, USA

Gary Goldberg Hunter Holmes McGuire Veterans Administration Medical Center, Department of Physical Medicine and Rehabilitation, Virginia Commonwealth University School of Medicine/Medical College of Virginia, Richmond, VA, USA

Myron Goldberg Department of Rehabilitation Medicine, University of Washington Medical Center, Seattle, WA, USA

Diane Cordry Golden Association of Assistive Technology Act Programs, Delmar, NY, USA

Charles J. Golden Center for Psychological Studies, Nova Southeastern University, Fort Lauderdale, FL, USA

Amy S. Goldman Association of Assistive Technology Act Programs (ATAP), Springfield, IL, USA 
Bram Goldstein Department of Gynecologic Oncology, Hoag Hospital Cancer Center, Newport Beach, CA, USA

Assawin Gongvatana Department of Psychiatry, University of California, San Diego, San Diego, CA, USA

Efrain Antonio Gonzalez College of Psychology, Nova Southeastern University, Fort Lauderdale, FL, USA

Utah State University, Logan, UT, USA

Matthew E. Goodwin Central Arkansas Veterans Healthcare System, Little Rock, AR, USA

Robert M. Gordon Rusk Rehabilitation, New York University Langone Medical Center, New York, NY, USA

Kimberly A. Gorgens Graduate School of Professional Psychology, University of Denver, Denver, CO, USA

Janet Grace Psychiatry and Human Behavior, Alpert Medical School of Brown University, Providence, RI, USA

Martin R. Graf Department of Neurosurgery, Virginia Commonwealth University Medical Center, Richmond, VA, USA

Lori Grafton Carolinas Rehabilitation Carolinas HealthCare System, Charlotte, NC, USA

Kristin M. Graham Department of Physical Medicine and Rehabilitation, Virginia Commonwealth University, Richmond, VA, USA

Michael R. Greher School of Medicine, Department of Neurosurgery, University of Colorado, Aurora, CO, USA

Sarah Griffin Virginia Commonwealth University, Richmond, VA, USA

Julie Griffith Department of Speech Pathology and Audiology, Ball State University, Muncie, IN, USA

Stephanie Griffiths Simon Fraser University, Burnaby, BC, Canada

Elizabeth S. Gromisch Psychology Service, VA Connecticut, West Haven, CT, USA

Benjamin Grover-Manthey Department of Physical Medicine and Rehabilitation, Indiana University, Indianapolis, IN, USA

William Guido Anatomy and Neurobiology, Virginia Commonwealth University Medical Center, Richmond, VA, USA

Desiree Gulliford Department of Psychology, University of Florida, Gainesville, FL, USA

Carly Gundrum Legal Psychology (Psychology and Law), Neuropsychology, Clinical Psychology, The Chicago School of Professional Psychology, Chicago, IL, USA 
Audrey H. Gutherie Rehabilitation Research and Development Center of Excellence Atlanta Veterans Administration Medical Center, Decatur, GA, USA

Karl Haberlandt Department of Psychology, Trinity College, Hartford, CT, USA

Martin Hahn Department of Biology, William Paterson University, Wayne, NJ, USA

Kathrine Hak Applied Psychology and Counselor Education, University of Northern Colorado, Greeley, CO, USA

Katherine Hallahan University of Cincinnati, Cincinnati, OH, USA

John Halperin Overlook Medical Center, Atlantic Health System, Summit, NJ, USA

Marla J. Hamberger Department of Neurology, Columbia University Medical Center, New York, NY, USA

Flora M. Hammond Department of Physical Medicine and Rehabilitation, Indiana University School of Medicine, Indianapolis, IN, USA

Benjamin Hampstead Department of Pharmacology, Virginia Commonwealth University, Richmond, VA, USA

Department of Psychiatry, University of Michigan, Michigan Alzheimer's Disease Center, Ann Arbor, MI, USA

Sarah Hannigen Department of Psychology, Allegheny Health Network, Pittsburgh, PA, USA

Stephanie L. Hanson College of Public Health and Health Professions, University of Florida, Gainesville, FL, USA

Keith Happawana Department of Psychology, William Paterson University, Wayne, NJ, USA

Janna L. Harris Department of Anatomy and Cell Biology, University of Kansas Medical Center, Kansas City, KS, USA

Hoglund Brain Imaging Center, University of Kansas Medical Center, Kansas City, KS, USA

Patti L. Harrison School Psychology, The University of Alabama, Tuscaloosa, AL, USA

Eric S. Hart University of Missouri Center for Health Care Quality, Columbia, MO, USA

Michael J. Hartman Department of Psychology, University of WisconsinMilwaukee, Milwaukee, WI, USA

Kari Hawkins Neuropsychology Department, Addenbrooke's Hospital, Cambridge, UK

Amy Heffelfinger Medical College of Wisconsin, Milwaukee, WI, USA 
Robert L. Heilbronner Chicago Neuropsychology Group, Chicago, IL, USA

Kenneth M. Heilman Department of Neurology, University of Florida College of Medicine, Center for Neurological Studies and the Research Service of the Malcom Randall Veterans Affairs Medical Center, Gainesville, FL, USA

Nancy Helm-Estabrooks Department of Communication Disorders and Sciences, College of Health and Human Sciences, Western Carolina University, Cullowhee, NC, USA

Nathan Henninger Department of Pediatrics, Nationwide Children's Hospital College of Medicine, Ohio State University, Columbus, OH, USA

Mary Hibbard Department of Rehabilitation Medicine, New York School of Medicine, New York, NY, USA

Ashley K. Hill Nationally Certified School Psychologist, Chicago, IL, USA

Tanisha G. Hill-Jarrett Division of Rehabilitation Neuropsychology, Department of Rehabilitation Medicine, Emory University, Atlanta, GA, USA

Yvonne Hindes Division of Applied Psychology, Faculty of Education, University of Calgary, Calgary, AB, Canada

Merrill Hiscock Department of Psychology, University of Houston, Houston, TX, USA

G. Alex Hishaw Neurology Section, Southern Arizona VA Healthcare System, Tucson, AZ, USA

Department of Neurology, University of Arizona, Tucson, AZ, USA

Elise K. Hodges Department of Psychiatry, Neuropsychology Division, University of Michigan Health System, Ann Arbor, MI, USA

Anna DePold Hohler Boston University Medical Center, Boston, MA, USA

Matthew H. Holcomb Department of Pharmacology, Virginia Commonwealth University, Richmond, VA, USA

Tracey Hollingsworth Developmental Assessment Program, Nationwide Children's Hospital, Columbus, OH, USA

Stephanie Hooker Department of Behavioral Sciences, Rush University Medical Center, Chicago, IL, USA

Kelly L. Hoover Department of Educational Psychology, Ball State University, Muncie, IN, USA

Crista A. Hopp Connected Pathways Coaching, Herndon, VA, USA

Karin F. Hoth Department of Medicine, University of Iowa, Iowa City, IA, USA

Gillian Hotz Department of Neurosurgery/The Miami Project to Cure Paralysis, University of Miami Miller School of Medicine, Miami, FL, USA 
Marianne Hrabok Department of Psychology, Addiction and Mental Health, Alberta Health Services, Edmonton, AB, Canada

Gabrielle Hromas Department of Clinical and Health Psychology, University of Florida, Gainesville, FL, USA

Leesa V. Huang Department of Psychology, California State University, Chico, CA, USA

Dawn H. Huber Department of Health Psychology, University of Missouri, Columbia, MO, USA

Adam Hudepohl Georgia State University, Atlanta, GA, USA

Trevor Huff Department of Psychology and Neuroscience Center, Brigham Young University, Provo, UT, USA

Bradley J. Hufford Neuropsychology, Rehabilitation Hospital of Indiana, Indianapolis, IN, USA

Candace Hughes School of Psychology, The Chicago School of Professional Psychology, Chicago, IL, USA

Joel W. Hughes Department of Psychology, Kent State University, Kent, $\mathrm{OH}, \mathrm{USA}$

David Hulac Department of School Psychology, College of Education and Behavioral Sciences, University of Northern Colorado, Greeley, CO, USA

Faiza Humayun Inpatient Traumatic Brain Injury Unit, James Haley VA Hospital, Tampa, FL, USA

Edward E. Hunter Department of Psychiatry and Behavioral Sciences, University of Kansas Medical Center, Kansas City, KS, USA

Scott J. Hunter Departments of Psychiatry and Behavioral Neuroscience, and Pediatrics, Pediatric Neuropsychology, Section of Child and Adolescent Psychiatry, The University of Chicago, Chicago, IL, USA

Julia Hussey Department of Psychology, University of Nevada, Las Vegas, Las Vegas, NV, USA

R. Matthew Hutchison Biogen Inc., Cambridge, MA, USA

Karen Hux Quality Living, Omaha, NE, USA

Summer Ibarra Rehabilitation Hospital of Indiana, Indianapolis, IN, USA

Naroa Ibarretxe-Bilbao Department of Methods and Experimental Psychology, Faculty of Psychology and Education, University of Deusto, Bilbao, Spain

Jean Ikanga Division of Rehabilitation Neuropsychology, Department of Rehabilitation Medicine, Emory University, Atlanta, GA, USA

Matilde Inglese Department of Neurology, Radiology and Neuroscience, Icahn School of Medicine at Mount Sinai, New York, NY, USA 
Farzin Irani Psychology Department, West Chester University of Pennsylvania, West Chester, PA, USA

Rubyat Islam Department of Psychology, Queens College of the City University of New York, Flushing, NY, USA

Peter K. Isquith Dartmouth Medical School, Lebanon, NH, USA

Cindy B. Ivanhoe Neurorehabilitation Specialists Baylor College of Medicine, The Institute for Rehabilitation and Research, Houston, TX, USA

Grant L. Iverson Department of Psychiatry, British Columbia Mental Health and Addictions, University of British Columbia, Vancouver, BC, Canada

Colleen E. Jackson VA Boston Healthcare System, Boston, MA, USA

Kimberle M. Jacobs Department of Anatomy and Neurobiology, Virginia Commonwealth University, Richmond, VA, USA

Mathew Jacobs Pennsylvania State University, University Park, PA, USA

Lisa A. Jacobson Department of Neuropsychology, Kennedy Krieger Institute, Baltimore, MD, USA

Department of Psychiatry and Behavioral Sciences, Johns Hopkins University School of Medicine, Baltimore, MD, USA

Kelly M. Janke Columbia University Medical Center, New York, NY, USA

Nicholas Jasinski Division of Neuropsychology, Henry Ford Health System, Detroit, MI, USA

Beth A. Jerskey Department of Psychiatry and Human Behavior, Alpert Medical School of Brown University, Providence, RI, USA

Amitabh Jha TBIMS National Data and Statistical Center, Craig Hospital, Englewood, CO, USA

Mi-Yeoung Jo Sherman Oaks, CA, USA

Joshua Johnson Department of Psychiatry (MC-2103), UConn Health Center, Farmington, CT, USA

Judy A. Johnson Special Education Department, Aldine Independent School District, Houston, TX, USA

Julene K. Johnson Center for Aging in Diverse Communities, University of California, San Francisco, San Francisco, CA, USA

Kristin L. Johnson Applied Psychology and Counselor Education, University of Northern Colorado, Greeley, CO, USA

Nancy Johnson Cognitive/Behavioral Neurology Center Northwestern Feinburg School of Medicine, Chicago, IL, USA

Susan K. Johnson Department of Psychology, University of North Carolina at Charlotte, Charlotte, NC, USA 
Taylor Johnson School of Psychology, The Chicago School of Professional Psychology, Chicago, IL, USA

Brick Johnstone Intrepid Spirit Center, Fort Belvoir, VA, USA

Robert D. Jones Department of Neurology, The University of Iowa, Iowa City, USA

Aaron N. Juni Neuropsychology Center of Maryland, LLC, Owings Mills, MD, USA

Solomon Kalkstein Psychiatry, University of Pennsylvania, Philadelphia, PA, USA

Randy W. Kamphaus College of Education, The University of Oregon, Eugene, OR, USA

Stephen M. Kanne Thompson Center for Autism and Neurodevelopmental Disorders, University of Missouri, Columbia, MO, USA

Edith Kaplan Department of Psychology, Suffolk University, Boston, MA, USA

Erica Kaplan Rehabilitation Medicine, Brain Injury Research Center, Icahn School of Medicine at Mount Sinai, New York, NY, USA

Paul E. Kaplan Capitol Clinical Neuroscience, Folsom, CA, USA

Richard F. Kaplan Department of Psychiatry (MC-2103), UConn Health Center, Farmington, CT, USA

Narinder Kapur Research Department of Clinical, Educational and Health Psychology, University College London, London, UK

Ninad Karandikar RAC, Department of PM\&R AND Physiatrist, Polytrauma Transitional Rehabilitation Program, VA Palo Alto, Palo Alto, California, USA

Department of Orthoedics, Stanford University, Palo Alto, California, USA

Stella Karantzoulis Modern Brain Center, New York, NY, USA

Nadine J. Kaslow Department of Psychiatry and Behavioral Sciences, Emory University School of Medicine, APA, Atlanta, GA, USA

Sheryl Katta-Charles Department of Physical Medicine and Rehabilitation, Indiana University School of Medicine, Indianapolis, IN, USA

Douglas I. Katz Department of Neurology, Boston University School of Medicine, Braintree, MA, USA

Michael Kaufman Department of Neurology, Carolinas Medical Center, Charlotte, NC, USA 
Margarita Kaushanskaya University of Wisconsin-Madison, Madison, WI, USA

Jacqueline M. Kawa Psychiatry and Behavioral Medicine, Children's Hospital of Wisconsin, Milwaukee, WI, USA

Jaeson Kaylegian Departments of Psychiatry and Behavioral Neuroscience, and Pediatrics, Pediatric Neuropsychology, Section of Child and Adolescent Psychiatry, The University of Chicago, Chicago, IL, USA

Neuropsychology Technician III, University of Chicago Medicine, Chicago, IL, USA

Jacob Kean Department of Physical Medicine and Rehabilitation, Indiana University School of Medicine, Indianapolis, IN, USA

C. Keith Conners Duke University Medical School, Durham, NC, USA

Avril J. Keller Alberta Children's Hospital, University of Calgary, Calgary, $\mathrm{AB}$, Canada

Kristy K. Kelly Educational Psychology, University of Wisconsin-Madison, Madison, WI, USA

Sally L. Kemp University of Missouri, Columbia, MO, USA

Thomas R. Kerkhoff College of Public Health and Health Professions, University of Florida, Gainesville, FL, USA

Kimberly A. Kerns Department of Psychology, University of Victoria, Victoria, BC, Canada

So Hyun Kim Yale Child Study Center, Yale School of Medicine, New Haven, CT, USA

Sangsun Kim Psychological Sciences, University of Missouri, Columbia, MO, USA

Sun Mi Kim Department of Psychology, Queens College of the City University of New York, Flushing, NY, USA

Tricia Z. King Department of Psychology and the Neuroscience Institute, Georgia State University, Atlanta, GA, USA

Brock Kirwan Psychology Department and the Neuroscience Center, Brigham Young University, Provo, UT, USA

Bonita P. "Bonnie" Klein-Tasman Department of Psychology, University of Wisconsin-Milwaukee, Milwaukee, WI, USA

Jennifer Sue Kleiner Department of Psychology, University of Arkansas for Medical Sciences Blandford Physician Center, Little Rock, AR, USA

Daniel W. Klyce Virginia Commonwealth University - School of Medicine, Richmond, VA, USA

C. Keith Conners: deceased. 
Samantha Knight Medicine, Royal College of Surgeons in Ireland, Dublin, Ireland

Kelly Knollman-Porter Department of Speech Pathology and Audiology, Miami University, Oxford, OH, USA

Tiffany Kodak Department of Psychology, University of Wisconsin-Milwaukee, Milwaukee, WI, USA

Julia Kolak Department of Psychology, William Paterson University, Wayne, NJ, USA

Stephanie A. Kolakowsky-Hayner Department of Rehabilitation Medicine, Icahn School of Medicine at Mount Sinai, New York, NY, USA

Dan Koonce The Chicago School of Psychology, Chicago, IL, USA

Paul T. Korte Harry S. Truman Memorial Veterans' Hospital, Columbia, MO, USA

Kathleen A. Koth Department of Psychiatry and Behavioral Medicine, Medical College of Wisconsin, Milwaukee, WI, USA

Zhifeng Kou Biomedical Engineering and Radiology, Wayne State University, Detroit, MI, USA

Rothem Kovner Department of Psychiatry, University of Wisconsin-Madison, Madison, WI, USA

Elizabeth Kozora Department of Medicine, National Jewish Medical and Research Center, National Jewish Health, Denver, CO, USA

Joel H. Kramer UCSF Memory and Aging Center UCSF Med Ctr, 09848AC, San Francisco, CA, USA

Matthew Kraybill Neuropsychology, Cottage Rehabilitation Hospital, Santa Barbara, CA, USA

Denise Krch Kessler Foundation, East Hanover, NJ, USA

Kate Krival Speech Pathology, School of Health Sciences, Edinboro University of Pennsylvania, Edinboro, PA, USA

Lauren B. Krupp Department of Neuropsychology Research, Stony Brook University SUNY Stony Brook, Stony Brook, NY, USA

Nuri Erkut Kucukboyaci Clinical Psychology, Rusk Institute - NYU Langone Health, New York, NY, USA

Beth Kuczynski Imaging of Dementia and Aging (IDeA) Laboratory, Department of Neurology and Center for Neuroscience, University of California, Davis, CA, USA

Jeffrey G. Kuentzel Wayne State University, Detroit, MI, USA

Richard Kunz Department of Physical Medicine and Rehabilitation, Virginia Commonwealth University, Richmond, VA, USA 
Brad Kurowski Department of Physical Medicine and Rehabilitation, Spaulding Rehabilitation Hospital, Massachusetts General Hospital, Brigham and Women's Hospital, Harvard Medical School, Cincinnati, OH, USA

Matthew M. Kurtz Department of Psychology, Wesleyan University, Middletown, CT, USA

Monica Kurylo Departments of Psychiatry and Rehabilitation Medicine, University of Kansas Medical Center, Kansas City, KS, USA

Christina Kwasnica Barrow Neurological Institute, Phoenix, AZ, USA

David Lachar University of Texas Houston Health Science Center, Houston, TX, USA

Susan Ladley Department of Physical Medicine and Rehabilitation, University of Colorado, Denver Health Medical Center, Denver, CO, USA

Ginette Lafleche Memory Disorders Research Center, VA Boston Healthcare System and Boston University School of Medicine, Boston, MA, USA

Audrey Lafrenaye Department of Anatomy and Neurobiology, Virginia Commonwealth University, Richmond, VA, USA

Sarah K. Lageman Parkinson's and Movement Disorders Center, Department of Neurology, School Of Medicine, Virginia Commonwealth University, Richmond, VA, USA

Melissa Lamar Rush Alzheimer's Disease Center, Chicago, IL, USA

Damon G. Lamb Department of Clinical and Health Psychology, University of Florida, Gainesville, FL, USA

Center for Cognitive Aging and Memory, McKnight Brain Institute, University of Florida, Gainesville, FL, USA

Department of Neurology, University of Florida, Gainesville, FL, USA

Brain Rehabilitation Research Center, Malcom Randall VAMC, Gainesville, FL, USA

Gudrun Lange Pain and Fatigue Study Center, New York, NY, USA

Mount Sinai Beth Israel Medical Center, New York, NY, USA

Rael T. Lange Defense and Veterans Brain Injury Center, Walter Reed National Military Medical Center, Bethesda, MD, USA

Karen G. Langer Department of Rehabilitation Medicine, Rusk Rehabilitation, NYU Langone Medical Center, New York, NY, USA

Kayla LaRosa Educational and Psychological Studies/TBI Model Systems, University of South Florida/J.A. Haley VA, Tampa, FL, USA

Michael J. Larson Brigham Young University, Provo, UT, USA

Jennifer C. G. Larson Department of Physical Medicine and Rehabilitation, University of Michigan, Ann Arbor, MI, USA 
Adele A. Larsson Department of Educational Psychology, Ball State University, Muncie, IN, USA

Javier Peña Lasa Department of Psychology, Deusto University, Bilbao, Spain

Thomas M. Laudate Department of Adult Neurology, Tufts Medical Center, Boston, MA, USA

Ronald M. Lazar Department of Neurology, University of Alabama at Birmingham, Birmingham, AL, USA

Victoria M. Leavitt Cognitive Neuroscience Division, Columbia University Medical School, New York, NY, USA

Sophie Lebrecht Visual Neuroscience Laboratory, Brown University, Providence, RI, USA

Kangmin D. Lee Department of Neurosurgery, Virginia Commonwealth University, Richmond, VA, USA

Sing Lee Department of Psychiatry, The Chinese University of Hong Kong, Shatin, Hong Kong SAR, China

Stacie A. Leffard Behavioral Medicine and Psychiatry, West Virginia University, Morgantown, WV, USA

George Leichnetz Virginia Commonwealth University, Richmond, VA, USA

Hoyle Leigh Department of Psychiatry, University of California, San Francisco, CA, USA

Sarah J. Leinen Institute for Graduate Clinical Psychology, Widener University, Chester, PA, USA

Jeannie Lengenfelder Kessler Foundation Research Center, West Orange, NJ, USA

José León-Carrión Human Neuropsychology Laboratory, School of Psychology, Department of Experimental Psychology, University of Seville, Seville, Spain

Center for Brain Injury Rehabilitation (CRECER), Seville, Spain

Umberto León-Domínguez Health Sciences Vice-Chancellor, Department of Psychology, University of Monterrey, Monterrey, Mexico

Human Neuropsychology Laboratory, School of Psychology, Department of Experimental Psychology, University of Seville, Seville, Spain

Pierre A. Leon Department of Psychology, William Paterson University, Wayne, NJ, USA

Vanesa C. Lerma Department of Neurological Surgery, Weill Cornell Medicine, New York, NY, USA

Holly Levin-Aspenson Department of Psychology, University of Notre Dame, Notre Dame, IN, USA 
Brian Levine Rotman Research Institute at Baycrest, Toronto, ON, Canada

Allen N. Lewis Jr. School of Health and Rehabilitation Science, University of Pittsburgh, Pittsburgh, PA, USA

Pamela H. Lewis Department of Rehabilitation Counseling, School of Allied Health Professions, Virginia Commonwealth University, Richmond, VA, USA

David J. Libon Departments of Geriatrics, Gerontology, and Psychology, Rowan University, New Jersey Institute for Successful Aging, School of Osteopathic Medicine, Stratford, NJ, USA

Edward Liebmann Department of Psychology, The University of Kansas, Lawrence, KS, USA

Tanya P. Lin Neurology Section, Southern Arizona VA Healthcare System, Tucson, AZ, USA

Department of Neurology, University of Arizona, Tucson, AZ, USA

Sara M. Lippa Defense and Veterans Brain Injury Center, Walter Reed National Military Medical Center, Bethesda, MD, USA

Hayley Loblein Department of Educational Psychology, The University of Texas at Austin, Austin, TX, USA

Dona Locke Psychiatry and Psychology, Mayo Clinic, Scottsdale, AZ, USA

Chris Loftis National Council for Community Behavioral Healthcare, Alexandria, VA, USA

Kenneth J. Logan Department of Speech, Language, and Hearing Sciences, University of Florida, Gainesville, FL, USA

Michelle Loman Department of Neurology, Medical College of Wisconsin, Milwaukee, WI, USA

Eduardo Lopez Rehabilitation Medicine, New York Medical College, Metropolitan Hospital, New York, NY, USA

Catherine Lord Center for Autism and the Developing Brain, New YorkPresbyterian Hospital/Westchester Division, White Plains, NY, USA

Janis Lorman School of Speech-Language Pathology and Audiology, The University of Akron, Akron, OH, USA

Rachel Losoff School Psychology, The Chicago School of Professional Psychology, Chicago, IL, USA

John A. Lucas Department of Psychiatry and Psychology, Mayo Clinic, Jacksonville, FL, USA

Stephen D. Luke National Dissemination Center for Children with Disabilities (NICHCY), Washington, DC, USA

Jacob T. Lutz Department of Special Education, Ball State University, Muncie, IN, USA 
Jon G. Lyon Mazomanie, WI, USA

Paige Lysne Department of Aging and Geriatric Research, Institute of Aging, University of Florida - College of Medicine, Gainesville, FL, USA

Kelly Teresa Macdonald Department of Psychology, University of Houston, Houston, TX, USA

Anna MacKay-Brandt Nathan S. Kline Institute for Psychiatric Research, Orangeburg, NY, USA

Taub Institute for Research on Alzheimer's Disease and the Aging Brain, Columbia University, New York, NY, USA

Stephanie Magou Department of Psychology, William Paterson University, Wayne, NJ, USA

E. Mark Mahone Department of Neuropsychology, Kennedy Krieger Institute, Baltimore, MD, USA

Department of Psychiatry and Behavioral Sciences, Johns Hopkins University School of Medicine, Baltimore, MD, USA

B. Makofske Legal Psychology (Psychology and Law), Neuropsychology, Clinical Psychology, Chicago School of Professional Psychology, Chicago, IL, USA

James F. Malec Department of Physical Medicine and Rehabilitation, Indiana University School of Medicine and the Rehabilitation Hospital of Indiana, Indianapolis, IN, USA

Amit X. Malhotra Department of Pediatric Specialty, Division of Neurology, TPMG, Kaiser Permanente East Bay Medical Center, Oakland, CA, USA

Paul Malloy Department of Psychiatry and Human Behavior, Brown University, Providence, RI, USA

William Victor Maloy The Virginia Institute of Pastoral Care, Richmond, VA, USA

Gail Malvestuto School of Psychology, The Chicago School of Professional Psychology, Chicago, IL, USA

Carlye B. G. Manna Department of Psychology, Queens College of the City University of New York (CUNY), Flushing, NY, USA

Bernice A. Marcopulos Department of Graduate Psychology, James Madison University, Harrisonburg, VA, USA

Christina R. Marmarou Neurosurgery, Virginia Commonwealth University, Richmond, VA, USA

Patrick Marsh Department of Psychiatry and Behavioral Sciences, University of South Florida College of Medicine, Tampa, FL, USA

Matthew P. Martens University of Missouri, Columbia, MO, USA 
Thomas Martin Center for Health Care Quality, University of MissouriColumbia, Columbia, MO, USA

Jairo Enrique Martinez Columbia University, New York, NY, USA

Guido Mascialino Department of Rehab Medicine Brain Injury Research, Mount Sinai School of Medicine, New York, NY, USA

Joshua M. Matyi Department of Psychology, Utah State University, Logan, UT, USA

Micah O. Mazurek Curry School of Education, University of Virginia, Charlottesville, VA, USA

Michèle M. M. Mazzocco Institute of Child Development, University of Minnesota, Minneapolis, MN, USA

David L. McCabe Department of Psychology, Rusk Rehabilitation, NYU Langone Medical Center, New York, NY, USA

Marissa McCarthy Department of Neurology, James A Haley Veterans Hospital, University of South Florida, Tampa, FL, USA

Rebecca McCartney Department of Behavioral Health, Kaiser Permenante, Atlanta, GA, USA

Rehabilitation Medicine, Emory University, Atlanta, GA, USA

Katherine S. McClellan Rehabilitation Research and Development Center, Atlanta Veterans Affairs Medical Center, Decatur, GA, USA

Dalene McCloskey Centennial Board of Cooperative Educational Services, Greeley, CO, USA

Erica McConnell Jefferson County Public Schools, School Psychologist, Golden, CO, USA

Michael McCrea Departments of Neurosurgery and Neurology, Medical College of Wisconsin, Milwaukee, WI, USA

Jacinta McElligott Rehabilitation Medicine, National Rehabilitation Hospital, Dun Laoghaire Co Dublin, Ireland

Melissa J. McGinn Anatomy and Neurobiology, Virginia Commonwealth University School of Medicine, Richmond, VA, USA

Pip McGirl The Chicago School of Professional Psychology, School Psychology Department, Chicago, IL, USA

Amanda McGovern Psychiatry, Columbia University Medical Center, New York, NY, USA

David E. McIntosh Department of Special Education, Teachers College, Bell State University, Muncie, IN, USA 
Miechelle McKelvey Department of Communication Disorders, COE B141, University of Nebraska Kearney, Kearney, NE, USA

Tamara McKenzie-Hartman Defense and Veterans Brain Injury Center, James A. Haley, VA Hospital, Tampa, FL, USA

Molly E. McLaren Center for Cognitive Aging and Memory, Department of Clinical and Health Psychology, University of Florida, Gainesville, FL, USA

Nicole C. R. McLaughlin Butler Hospital Alpert Medical School of Brown University, Providence, RI, USA

Brian T. McMahon Department of Rehabilitation Counseling, Virginia Commonwealth University, Richmond, VA, USA

Lemmietta McNeilly Speech-Language Pathology, American Speech-Language-Hearing Association, Rockville, MD, USA

Rory McQuiston Anatomy and Neurobiology, Virginia Commonwealth University, Richmond, VA, USA

Linda McWhorter Department of Psychology, University of North Carolina at Charlotte, Charlotte, NC, USA

Mary-Ellen Meadows Division of Cognitive and Behavioral Neurology, Brigham and Women's Hospital, Boston, MA, USA

Stephanie Mears MHS Inc, Toronto, ON, Canada

Michael S. Mega Center for Cognitive Health, Portland, OR, USA

Stephen S. Meharg Center for Memory and Learning, Longview, WA, USA

Erica P. Meltzer Department of Psychology, Queens College and The Graduate Center of the City University of New York, Flushing, NY, USA

Patricia Melville Department of Neuropsychology Research, Stony Brook University SUNY Stony Brook, Stony Brook, NY, USA

Andrew Menatti Neuropsychology, University of Missouri, Columbia, MO, USA

John E. Mendoza Department of Psychiatry and Neuroscience, Tulane Medical School and SE Louisiana Veterans Healthcare System, New Orleans, LA, USA

Mark Mennemeier Neurobiology and Developmental Sciences, University of Arkansas for Medical Sciences, Little Rock, AR, USA

Randall E. Merchant Department of Anatomy and Neurobiology, Virginia Commonwealth University Medical Center, Richmond, VA, USA

Brad Merker Henry Ford Health Systems, Detroit, MI, USA

Carolyn B. Mervis Department of Psychological and Brain Sciences, University of Louisville, Louisville, KY, USA 
Gary B. Mesibov University of North Carolina at Chapel Hill, Chapel Hill, NC, USA

Linda A. Meyer Speech Therapy, Augusta Health, Waynesboro, VA, USA

John E. Meyers Department of Neuropsychology, Comprehensive Medpsych Systems, Sarasota, FL, USA

David Michalec Division of Psychology Ohio State University Nationwide Children's Hospital, Developmental Assessment Program, Columbus, OH, USA

Jackie L. Micklewright Georgia State University, Department of Psychology and the Neuroscience Institute, Atlanta, GA, USA

Christine J. Mihaila Northeast Regional Epilepsy Group, New York, NY, USA

Eric N. Miller Palm Springs, CA, USA

Ana Mills Department of Physical Medicine and Rehabilitation, Virginia Commonwealth University, Richmond, VA, USA

Ginger Mills Graduate Institute of Professional Psychology, University of Hartford, West Hartford, CT, USA

Brenda Atkinson Milner Montreal Neurological Institute and Hospital, Montreal, QC, USA

Kristin Moffett Emory University, Atlanta, GA, USA

Ethan Moitra Department of Psychiatry and Human Behavior, Brown University, Providence, RI, USA

Doris S. Mok Department of Psychology, Faculty of Social Sciences and Humanities University of Macau, Taipa, Macau SAR, China

Ashley Mondragon Department of Psychology, William Paterson University, Wayne, NJ, USA

Anna Bacon Moore Department of Rehabilitation Medicine, Division of Neuropsychology, Emory University School of Medicine, Atlanta, GA, USA

Brittney Moore Department of Educational Psychology, Ball State University, Muncie, IN, USA

Lisa Moran Department of Psychology, Nationwide Children's Hospital, Columbus, $\mathrm{OH}$, USA

Joseph E. Mosley Department of Psychology, William Paterson University, Wayne, NJ, USA

Margaret Moult Olin Neuropsychiatry Research Center, Institute of Living, Hartford, CT, USA

Martin Mrazik Department of Educational Psychology, University of Alberta, Edmonton, AB, Canada 
Christine Mullen Emory Health Care, Atlanta, GA, USA

Courtney Murphy Belmont Behavioral Hospital, Philadelphia, PA, USA

Mary Pat Murphy MSN, CRRN, Paoli, PA, USA

Suzanne Musil Rush University Medical Center, Chicago, IL, USA

Charlsie Myers Department of Social Sciences, Coastal College of Georgia, Brunswick, GA, USA

Sylvie Naar-King Detroit, MI, USA

Risa Nakase-Richardson Mental Health and Behavioral Sciences, Department of Medicine, James A. Haley Veterans Hospital, University of South Florida, Defense and Veterans Brain Injury Center, Center of Innovation in Disability and Rehabilitation Research, Tampa, FL, USA

Luba Nakhutina Department of Neurology, SUNY Downstate Medical Center, Brooklyn, NY, USA

Madison Neirmeyer Department of Psychology, The University of Utah, Salt Lake City, UT, USA

Robi L. Nelson Department of Psychiatry and Behavioral Sciences, University of South Florida College of Medicine, Tampa, FL, USA

Department of Pharmacology, Virginia Commonwealth University, Richmond, VA, USA

Aaron P. Nelson Center for Brain Mind Medicine, Brigham and Women's Hospital and Harvard Medical School, Boston, MA, USA

Christina Nessler VA Salt Lake City Health Care System, Salt Lake City, UT, USA

Robert Newby Division of Neurology and Pediatrics, Medical College of Wisconsin, Milwaukee, WI, USA

Paul Newman Department of Medical Psychology and Neuropsychology, Drake Center, Cincinnati, OH, USA

Christine Maguth Nezu Department of Psychology, Drexel University-Hahnemann Campus, Philadelphia, PA, USA

Louisa Ng Department of Rehabilitation Medicine, Royal Melbourne Hospital, Parkville, VIC, Australia

Hien Nguyen Department of Pediatrics, Kaiser Permanente East Bay, Oakland, CA, USA

Jody S. Nicholson Department of Psychology, University of Notre Dame, Notre Dame, IN, USA

Jared A. Nielsen Departments of Psychiatry and Psychology, Harvard University and Massachusetts General Hospital, Cambridge, MA, USA 
Janet P. Niemeier Carolinas Rehabilitation, Carolinas Healthcare System, Charlotte, NC, USA

Madison Niermeyer Department of Psychology, Clinical Psychology, The University of Utah, Salt Lake City, UT, USA

Christine Nieves Robert Wood Johnson Medical School, Rutgers University, Piscataway, NJ, USA

C. Michael Nina Department of Psychology, William Paterson University, Wayne, NJ, USA

Ignatius Nip School of Speech, Language, and Hearing Sciences, San Diego State University, San Diego, CA, USA

Nicole R. Nissim Department of Clinical and Health Psychology College of Public Health and Health Professions, Center for Cognitive Aging and Memory, McKnight Brain Institute (Primary), University of Florida, Gainesville, FL, USA

Department of Neuroscience, University of Florida, Gainesville, FL, USA

Natalie O. Nordlund Legal Psychology (Psychology and Law), Neuropsychology, Clinical Psychology, The Chicago School of Professional Psychology, Chicago, IL, USA

David Nordstokke Werklund School of Education, University of Calgary, Calgary, AB, Canada

Virginia A. Norris San Quentin State Prison, San Quentin, CA, USA

Olga Noskin Neurology Group of Bergen County, P.A, Ridgewood, NJ, USA

Thomas A. Novack Department of Physical Medicine and Rehabilitation, University of Alabama at Birmingham, Birmingham, AL, USA

Alicia Nuñez Department of Psychology, University of Nevada, Las Vegas, NV, USA

Meena Nuthi University of Florida, Gainesville, FL, USA

Andrew O'Shea Department of Aging and Geriatric Research, College of Medicine, Institute on Aging, University of Florida, Gainesville, FL, USA

Deirdre M. O'Shea Department of Clinical and Health Psychology, Center for Cognitive Aging and Memory, University of Florida, Gainesville, FL, USA

Kathleen O'Toole Children's Healthcare of Atlanta, Atlanta, GA, USA

Thomas Oakland Department of Educational Psychology, College of Education University of Florida, Gainesville, FL, USA 
Christa Ochoa Center for Cognitive Aging and Memory, Department of Clinical and Health Psychology, University of Florida, Gainesville, Florida, USA

Natalia Ojeda Department of Methods and Experimental Psychology, Faculty of Psychology and Education, University of Deusto, Bilbao, Spain

Jonathan A. Oler Department of Psychiatry, University of Wisconsin-Madison, Madison, WI, USA

Traci W. Olivier Neuropsychology Section, Department of Psychology, St. Jude Children's Research Hospital, Memphis, TN, USA

Katie Osborn Florida School of Professional Psychology at Argosy University, Tampa, FL, USA

Zachary H. Osborn Behavioural Health Service Line, Harry S. Truman Memorial Veteran's Hospital, Columbia, MO, USA

Celita J. Owens Immaculata University, Immaculata, PA, USA

Matthew J. L. Page Allegheny General Hospital, Pittsburgh, PA, USA

Psychology, Allegheny Health Network, Pittsburgh, PA, USA

Rohan Palmer Institute for Behavioral Genetics, University of Colorado at Boulder, Boulder, CO, USA

Christina A. Palmese Department of Neurology, Mount Sinai Beth Israel, New York, NY, USA

Teresa Palumbo Administration Communications Senior University Relations Specialist, University of Wisconsin-Madison Waisman Center, Madison, WI, USA

Juhi Pandey Department of Psychology, University of Connecticut, Storrs, CT, USA

Carlos Bo Pang Department of Psychology, Faculty of Social Sciences and Humanities, University of Macau, Macao, Macau SAR, China

Aimilia Papazoglou Department of Psychology and the Neuroscience Institute, Georgia State University, Atlanta, GA, USA

Kathryn V. Papp Department of Psychology, The University of Connecticut, Storrs, CT, USA

Rick Parente Department of Psychology, Towson University, Towson, MD, USA

John Parkhurst Psychiatry and Behavioral Medicine, Children's Hospital of Wisconsin, Medical College of Wisconsin, Milwaukee, WI, USA

Matthew R. Parry Neurosurgery, Virginia Commonwealth University, Richmond, VA, USA

Lisa A. Pass Department of Educational Psychology, Ball State University, Muncie, IN, USA 
Nicholas Pastorek Rehabilitation and Extended Care Line, Michael E. DeBakey VA Medical Center, Houston, TX, USA

Janet P. Patterson Audiology and Speech-Language Pathology Service, VA Northern California Health Care System, Martinez, CA, USA

Diane Paul Clinical Issues in Speech-Language Pathology, American Speech-Language-Hearing Association, Rockville, MD, USA

Nina Paul Department of Psychology, University of Nevada-Las Vegas, Las Vegas, Nevada, USA

Rebecca Pavlick Department of Psychology, William Paterson University, Wayne, NJ, USA

Shelley Pelletier Board Certified in School Psychology, Shoreline Pediatric Neuropsychology Services, LLC, Old Saybrook, CT, USA

Javier Peña Department of Methods and Experimental Psychology, Faculty of Psychology and Education, University of Deusto, Bilbao, Spain

Suzanne Penna Department of Rehabilitation Medicine, Emory University School of Medicine, Atlanta, GA, USA

Dana L. Penney Department of Neurology, The Lahey Clinic, Burlington, MA, USA

Molly Penzenik Rocky Mountain Mental Illness Research Education and Clinical Center, Denver, CO, USA

University of Colorado, Anschutz Medical Campus, Aurora, CO, USA

Alexandra Perrault University of Cincinnati, Cincinnati, OH, USA

Lexi Perrault University of Cincinnati, Cincinnati, OH, USA

Paul B. Perrin Department of Psychology, Virginia Commonwealth University, Richmond, VA, USA

Kenneth R. Perrine Neurological Surgery, Weill Cornell Medicine, New York, NY, USA

Amy Peterman Department of Psychological Science, University of North Carolina at Charlotte, Charlotte, NC, USA

Maria Petracca Department of Neurology, Icahn School of Medicine at Mount Sinai, New York, NY, USA

Jo Ann Petrie Department of Psychology and the Neuroscience Center, Brigham Young University, Provo, UT, USA

LeAdelle Phelps University at Buffalo, State University of New York, Buffalo, NY, USA

Angela M. Philippus Craig Hospital, Englewood, CO, USA

Linda L. Phillips Anatomy and Neurobiology, Virginia Commonwealth University, Richmond, VA, USA 
Jesse J. Piehl Department of Pharmacology, Virginia Commonwealth University, Richmond, VA, USA

Elizabeth I. Pierpont Department of Pediatrics, Division of Clinical Behavioral Neuroscience, University of Minnesota, Minneapolis, MN, USA

Eric E. Pierson Department of Educational Psychology, Ball State University, Muncie, IN, USA

Irene Piryatinsky Butler Hospital and Alpert Medical School of Brown University, Providence, RI, USA

Jenni Pitkanen Product Development at MHS Inc., Toronto, ON, Canada

Kenneth Podell Houston Methodist Hospital, Houston, TX, USA

Henry Ford Health Systems, Detroit, MI, USA

Ben Polakoff Department of Educational Psychology, The University of Utah, Salt Lake City, UT, USA

Elena Polejaeva Department of Clinical and Health Psychology, University of Florida, Gainesville, FL, USA

Donna Polelle Communication Sciences and Disorders, College of Science and Mathematics, University of South Florida Sarasota-Manatee, Sarasota, FL, USA

Eric S. Porges Department of Clinical and Health Psychology, University of Florida, Gainesville, FL, USA

Center for Cognitive Aging and Memory, McKnight Brain Institute, University of Florida, Gainesville, FL, USA

Department of Neurology, University of Florida, Gainesville, FL, USA

Matthew R. Powell Division of Neurocognitive Disorders, Department of Psychiatry and Psychology, Mayo Clinic, Rochester, MN, USA

Tiffany L. Powell Department of Neurosurgery, Virginia Commonwealth University, Richmond, VA, USA

Elizabeth Power Legal Psychology (Psychology and Law), Neuropsychology, Clinical Psychology, Chicago School of Professional Psychology, Chicago, IL, USA

The College of Saint Rose, Albany, NY, USA

Natalia Ojeda Del Pozo Fundamentals and Methods of Psychology, University of Deusto, Bilbao, Spain

Victor R. Preedy Faculty of Life Sciences and Medicine, King's College London, London, UK

Andrew Preston Department of Pediatrics, Chapel Hill Pediatric Psychology, Chapel Hill, NC, USA

Catherine C. Price Department of Clinician and Health Psychology and Dept of Anesthesiology, University of Florida, Florida, USA 
George P. Prigatano Department of Clinical Neuropsychology, Barrow Neurological Institute, St. Joseph's Hospital and Medical Center, Phoenix, AZ, USA

Michelle Ann Prosje NeuroBehavioral Specialists of Jacksonville, Inc., Jacksonville, FL, USA

Antonio E. Puente Depatment of Psychology, University of North Carolina Wilmington, Wilmington, NC, USA

Anneliese Radke Department of Neurology, University of California, Davis, CA, USA

Susie Engi Raiford Assessment and Instruction, Pearson, San Antonio, TX, USA

Robert D. Rainer Department of Psychology, University of Florida, Gainesville, FL, USA

Vanessa L. Ramos Scarborough Department of Neuropsychology, Kennedy Krieger Institute, Baltimore, MD, USA

Kate D. Randall Department of Psychology, University of Victoria, Victoria, BC, Canada

Steven Z. Rapcsak Neurology Section, Southern Arizona VA Healthcare System, Tucson, AZ, USA

Department of Neurology, University of Arizona, Tucson, AZ, USA

Sarah A. Raskin Department of Psychology and Neuroscience Program, Trinity College, Hartford, CT, USA

Joseph F. Rath NYU Langone Medical Center, Department of Psychology, Rusk Institute of Rehabilitation Medicine, New York, NY, USA

Holly Rau Department of Psychology, The University of Utah, Salt Lake City, UT, USA

Anastasia Raymer Communication Disorders and Special Education, Old Dominion University, Norfolk, VA, USA

Jennifer Linton Reesman Kennedy Krieger Institute/Johns Hopkins University School of Medicine, Baltimore, MD, USA

Christine Reid Department of Rehabilitation Counseling, Virginia Commonwealth University, Richmond, VA, USA

Stephanie A. Reid-Arndt School of Health Professions - Health Psychology, University of Missouri, Columbia, MO, USA

Sheryl Reminger Psychology Department, University of Illinois at Springfield, Springfield, IL, USA

Kathryn K. Reva University of Northern Colorado, New York, NY, USA

Jose A. Rey College of Pharmacy, Nova Southeastern University, Ft. Lauderdale, FL, USA 
Cecil R. Reynolds Texas A\&M University, College Station, TX, USA

Jill B. Rich Department of Psychology, York University, Toronto, ON, Canada

Lindsey Richards Department of Clinical and Health Psychology, Center for Cognitive Aging and Memory, University of Florida, Gainesville, FL, USA

Robert Rider Department of Psychology, Drexel University, Philadelphia, PA, USA

Giulia Righi Visual Neuroscience Laboratory, Brown University, Providence, RI, USA

Diana L. Robins AJ Drexel Autism Institute, Drexel University, Philadelphia, PA, USA

Tresa Roebuck-Spencer Jefferson Neurobehavioral Group, Metairie, LA, USA

Daniel E. Rohe Mayo Clinic, Rochester, MN, USA

Cynthia Rolston Department of PM\&R, Virginia Commonwealth University-Medical College of Virginia, Richmond, VA, USA

Maryellen Romero Department of Psychiatry and Behavioral Sciences, Tulane University School of Medicine, New Orleans, LA, USA

Katherine A. Roof Department of Psychology, University of North Carolina at Charlotte, Charlotte, NC, USA

Susan Ropacki VA Palo Alto, Polytrauma System of Care, Polytrauma Transitional Rehabilitation Program, VA Palo Alto Health Care System, Palo Alto, California, USA

Neurosurgery, Stanford University, Palo Alto, California, USA

Jon Rose Spinal Cord Injury Clinic, Veterans Affairs Palo Alto Healthcare System, Palo Alto, CA, USA

Carole R. Roth Otolaryngology Clinic, Speech Division, Naval Medical Center, San Diego, CA, USA

Elliot J. Roth Department of Physical Medicine and Rehabilitation, Northwestern University, Feinberg School of Medicine, Chicago, IL, USA

Robert M. Roth Geisel School of Medicine at Dartmouth / DHMC, Lebanon, NH, USA

Linda Rowley Waisman Center Family Village, University of Madison, Madison, WI, USA

Donald R. Royall Deptartment of Psychiatry, The University of Texas Health Center at San Antonio, San Antonio, TX, USA

Shahal Rozenblatt Advanced Psychological Assessment P. C., Smithtown, NY, USA 
Alexandra Rudd-Barnard One Neuro, West Los Angeles, California, USA

Ronald Ruff San Francisco Clinical Neurosciences, University of California, San Francisco, CA, USA

Jessica Somerville Ruffolo Department of Psychology, University of Washington, Seattle, Washington, DC, USA

Ruba Rum College of Medicine Psychiatry and Behavioral Neurosciences, University of South Florida, Tampa, FL, USA

Anthony C. Ruocco Department of Psychology, University of Toronto, Toronto, ON, Canada

Beth Rush Psychiatry and Psychology, Mayo Clinic, Jacksonville, FL, USA

Michele Rusin Emory University/Rehabilitation Medicine, Atlanta, GA, USA

Julia Rutenberg Rehabilitation Medicine, Emory University, Atlanta, GA, USA

John P. Ryan Department of Psychiatry, University of Pittsburgh, Pittsburgh, PA, USA

Bruce Rybarczyk Department of Psychology, Virginia Commonwealth University, Richmond, VA, USA

Catherine M. Rydell American Academy of Neurology, Minneapolis, MN, USA

Sara R. Rzepa Clinical, Education, and Public Safety at MHS Inc, Toronto, ON, Canada

Bonnie C. Sachs Parkinson's and Movement Disorders Center, Virginia Commonwealth University, Richmond, VA, USA

Amanda L. Sacks-Zimmerman Department of Neurological Surgery, Weill Cornell Medicine, New York, NY, USA

Donald H. Saklofske Department of Psychology, University of Western Ontario, London, ON, Canada

Christina Salama Kennedy Krieger Institute/Johns Hopkins University School of Medicine, Baltimore, MD, USA

Christine M. Salinas Space Coast Neuropsychology Center, Melbourne, FL, USA

Stephanie L. Salinas Department of Rehabilitation Medicine, The Georgia School of Professional Psychology at Argosy University-Atlanta, Atlanta, GA, USA

Stephen P. Salloway Butler Hospital Alpert Medical School of Brown University, Providence, RI, USA

Jeffery Samuels Inpatient Rehabilitation Unit, North Broward Medical Center, Deerfield Beach, FL, USA 
Orlando Sánchez Minneapolis VA Health Care System, Minneapolis, MN, USA

Mark A. Sandberg Neuropsychology, Northport VA Medical Center, Smithtown, NY, USA

Chelsea Sanders Department of Psychology, Utah State University, Logan, UT, USA

R. Sands Legal Psychology (Psychology and Law), Neuropsychology, Clinical Psychology, The Chicago School of Professional Psychology, Chicago, IL, USA

Marla Sanzone Independent Practice, Loyola College of Maryland, Annapolis, MD, USA

Celine A. Saulnier Department of Pediatrics, Emory University School of Medicine, Atlanta, GA, USA

Lynn A. Schaefer Physical Medicine and Rehabilitation, Nassau University Medical Center, East Meadow, NY, USA

Philip Schatz Department of Psychology, Saint Joseph's University, Philadelphia, PA, USA

Mike R. Schoenberg Department of Psychiatry and Behavioral Sciences, University of South Florida College of Medicine, Tampa, FL, USA

Aaron Schrader Applied Psychology and Counselor Education, University of Northern Colorado, Greeley, CO, USA

William A. Schraegle Pediatric Neuropsychology, Dell Children's Medical Center of Central Texas, Austin, TX, USA

Department of Educational Psychology, The University of Texas at Austin, Austin, TX, USA

Jillian Schuh Department of Psychology, University of Connecticut, Storrs, CT, USA

Christian Schutte Henry Ford Allegiance, Jackson, MI, USA

John D. Dingell VA Medical Center, Psychology Section (11MHPS), Detroit, MI, USA

Kerri A. Scorpio Department of Psychology, Queens College of the City University of New York, Flushing, NY, USA

Paige Seegan Clinical Psychology Program, Texas Tech University, Lubbock, TX, USA

Peter W. Seely Department of Pharmacology, Virginia Commonwealth University, Richmond, VA, USA

Daniel L. Segal Department of Psychology, University of Colorado at Colorado Springs, Colorado Springs, CO, USA 
Talia R. Seider Department of Clinical and Health Psychology, College of Public Health and Health Professions, University of Florida, Gainesville, FL, USA

Center for Cognitive Aging and Memory, McKnight Brain Institute, University of Florida, Gainesville, FL, USA

Robin Sekerak Waikato District Health Board, Hamilton, New Zealand

Svetlana Serova Department of Rehabilitation Medicine, Mount Sinai School of Medicine, New York, NY, USA

Laura Shank Rehabilitation Psychology and Neuropsychology, Physical Medicine and Rehabilitation University of Michigan, Ann Arbor, MI, USA

Casey R. Shannon University of Northern Colorado, Greeley, CO, USA

Anuj Sharma Virginia Commonwealth University School of Medicine, Richmond, VA, USA

Rhonna Shatz Department of Neurology and Rehabilitation, MED-Neurology, University of Cincinnati, Cincinnati, OH, USA

Bennett A. Shaywitz Department of Pediatrics, Yale University School of Medicine, New Haven, CT, USA

Sally E. Shaywitz Department of Pediatrics, Yale University School of Medicine, New Haven, CT, USA

Victoria Shea University of North Carolina at Chapel Hill, Chapel Hill, NC, USA

Judith A. Shechter Wynnewood, PA, USA

Tamara Goldman Sher Institute of Psychology, Illinois Institute of Technology, Chicago, IL, USA

Elisabeth M. S. Sherman Copeman Healthcare Centre, Calgary, AB, Canada

Cynthia X. Shi Emory College at Emory University, Atlanta, GA, USA

Cheryl L. Shigaki Department of Health Psychology, University of Missouri, Columbia, MO, USA

Lindsay C. Shima Department of Psychology, Neuropsychology Research Group, West Chester University of Pennsylvania, West Chester, PA, USA

Gerald Showalter Department of Psychiatry and Neurobehavioral Sciences, University of Virginia School of Medicine, Charlottesville, VA, USA

Seema Shroff Department of Anatomy and Neurobiology, Virginia Commonwealth University, Richmond, VA, USA

David Ho Keung Shum Griffith University, School of Psychology, Mt Gravatt Campus Griffith University, Brisbane, QLD, Australia 
Melissa Shuman-Paretsky Department of Rehabilitation Medicine, Mount Sinai School of Medicine, New York, NY, USA

Linda Shuster Interdisciplinary Health Sciences Ph.D. Program and Department of Speech, Language, and Hearing Sciences, Western Michigan University, Kalamazoo, MI, USA

Kevin Sickinger Chronic Effects of Neurotrauma Consortium (CENC), Virginia Commonwealth University, Richmond, VA, USA

Marc A. Silva Mental Health and Behavioral Sciences Service, James A. Haley Veterans' Hospital, Tampa, FL, USA

Preeti Sinha Department of Clinical and Health Psychology, University of Florida, Gainesville, FL, USA

Sue Ann Sisto School of Health Technology and Management Stony Brook University, Stony Brook, NY, USA

Gill Sitarenios Multi-Health Systems Inc., Toronto, ON, Canada

Ketharini Sivasegaran Whitby Vision Care, Whitby, ON, Canada

Amanda Skierkiewicz School Psychology, The Chicago School of Professional Psychology, Chicago, IL, USA

Beth Slomine Johns Hopkins University School of Medicine, Baltimore, MD, USA

Audrey Smerbeck School and Educational Psychology, University at Buffalo, The State University of New York, Buffalo, NY, USA

Daniel Smith Department of Psychology, Drexel University, Philadelphia, PA, USA

Winship Cancer Institute, Emory University, Atlanta, GA, USA

Kristen Smith Department of Psychology and the Neuroscience Institute, Georgia State University, Atlanta, GA, USA

Marian L. Smith Via Christi Behavioral Health Crossroads Counseling Center, Via Christi Hospital Pittsburg Mt. Carmel, Pittsburg, KS, USA

Nicholas David Smith Educational and Psychological Studies, University of South Florida/TBI Model Systems, James A. Haley VA Hospital, Tampa, FL, USA

Lucia Smith-Wexler Rusk Rehabilitation, New York University Langone Health, New York, NY, USA

Kayle E. Sneed Department of Communication Sciences and Disorders, The University of Oklahoma Health Sciences Center, Oklahoma City, OK, USA

Jill Snyder Behavioral Health Services, Boston Public Schools, Roxbury, MA, USA 
McKay Moore Sohlberg Communication Disorders and Sciences, University of Oregon, Eugene, OR, USA

Carney Sotto College of Allied Health Sciences, University of Cincinnati, Cincinnati, $\mathrm{OH}$, USA

Barbara Spacca Anna Meyer Children's Hospital, Florence, Italy

Sara S. Sparrow Yale University Child Study Center, New Haven, CT, USA

Ferrinne Spector Psychology, Edgewood College, Madison, WI, USA

April Spivack Department of Management and Human Resources, University of Wisconsin Oshkosh, Oshkosh, WI, USA

Beth Springate Department of Psychiatry, University of Connecticut Health Center, Farmington, CT, USA

Maria St. Pierre Department of Psychology, Towson University, Towson, MD, USA

Vess Stamenova Rotman Research Institute at Baycrest, Toronto, ON, Canada

Amy J. Starosta Departments of Psychiatry and Physical Medicine and Rehabilitation, University of Colorado Denver, Aurora, CO, USA

Susan Steffani CCC-SLP, Department of Communication Sciences and Disorders, California State University, Chico, Chico, CA, USA

Taryn M. Stejskal Department of Physical Medicine and Rehabilitation, Virginia Commonwealth University Medical Center, Virginia, VA, USA

Jeremy Stevenson Department of Family Medicine, University of Kansas Medical Center, Kansas City, KS, USA

William Stiers Johns Hopkins University School of Medicine, Baltimore, MD, USA

Jordan Stiver UCSF Memory and Aging Center, University California San Francisco, San Francisco, CA, USA

Esther Strauss Department of Psychology, University of Victoria, Victoria, BC, Canada

Anthony Y. Stringer Department of Rehabilitation Medicine, Emory University, Atlanta, GA, USA

Donald T. Stuss University of Toronto, Toronto, ON, Canada

Sunnybrook Health Sciences Centre, Toronto, ON, Canada

Rotman Research Institute of Baycrest, Toronto, ON, Canada

Lauren Stutts Department of Health and Human Values, Davidson College, Davidson, NC, USA

Yana Suchy Department of Psychology, The University of Utah, Salt Lake City, UT, USA 
Joyce Suh Kennedy Krieger Institute/Johns Hopkins University School of Medicine, Baltimore, MD, USA

James F. Sumowski Teachers College Columbia University, New York, NY, USA

Dong Sun Department of Aantomy and Neurobiology, School of Medicine, Virginia Commonwealth University Medical Center, Richmond, VA, USA

Uma Suryadevara Department of Psychiatry, University of Florida, Gainesville, FL, USA

Zoë N. Swaine Keele University, Keele, Newcastle ST5 5BG, UK

Joan Swearer Department of Neurology, University of Massachusetts Medical School, Worcester, MA, USA

Lawrence H. Sweet Department of Psychology, University of Georgia, Athens, GA, USA

Rod Swenson Department of Psychiatry and Behavioral Science, University of North Dakota School of Medicine, Fargo, ND, USA

Russell H. Swerdlow University of Kansas School of Medicine, Landon Center on Aging, Kansas City, KS, USA

Sarah M. Szymkowicz Department of Clinical and Health Psychology, College of Public Health and Health Professions, University of Florida, Gainesville, FL, USA

Jing Ee Tan Division of Neurology, University of British Columbia, Vancouver, BC, Canada

Vancouver General Hospital, Vancouver, BC, Canada

Department of Psychology, University of Victoria, Victoria, BC, Canada

Michael J. Tarr Visual Neuroscience Laboratory, Brown University, Providence, RI, USA

David F. Tate Brain Imaging and Behavior Laboratory, Missouri Institute of Mental Health (MIMH), University of Missouri, St. Louis (UMSL), Berkeley, MO, USA

Ella B. Teague Department of Neurorehabilitation, Reykjalundur Rehabilitation Center, Mosfellsbær, Iceland

Richard Temple Clinical Operations, CORE Health Care, Dripping Springs, TX, USA

Claire Thomas-Duckwitz University of Northern Colorado, Greeley, CO, USA

Cynthia K. Thompson Northwestern University, Evanston, IL, USA 
Jacob W. Tickle School of Psychology, The Chicago School of Professional Psychology, Chicago, IL, USA

Jennifer Tinker Department of Neurology, Thomas Jefferson University, Philadelphia, PA, USA

Michelle Marie Tipton-Burton Physical Medicine and Rehabilitation, Santa Clara Valley Medical Center, San Jose, CA, USA

Jeffrey B. Titus Comprehensive Epilepsy Program, Dell Children's Medical Center of Central Texas, Austin, TX, USA

Department of Psychology, The University of Texas at Austin, Austin, TX, USA

Teri A. Todd California State University, Northridge, Northridge, CA, USA

Nam Tran Neurosurgery, Virginia Commonwealth University Medical Center, Richmond, VA, USA

Alexander I. Tröster Department of Clinical Neuropsychology and Center for Neuromodulation, Barrow Neurological Institute, Phoenix, AZ, USA

Daniel Tranel Department of Neurology, The University of Iowa, Iowa City, USA

Angela K. Troyer Neuropsychology and Cognitive Health Program, Baycrest Centre for Geriatric Care, Toronto, ON, Canada

Tina Trudel Northeast Evaluation Specialists, PLLC, Dover, DE, USA

William Tsang Department of Psychology, William Paterson University, Wayne, NJ, USA

Theodore Tsaousides Department of Rehab Medicine Brain Injury Research, Mount Sinai School of Medicine, New York, NY, USA

JoAnn Tschanz Department of Psychology, Utah State University, Logan, UT, USA

Center for Epidemiologic Studies, Utah State University, Logan, UT, USA

Jessica Tsou Department of Psychiatry, University of Kansas Medical Center, Kansas City, KS, USA

Lyn S. Turkstra School of Rehabilitation Science, McMaster University, Hamilton, ON, Canada

Margaret Tuttle Department of Psychiatry, Massachusetts General Hosptial, Boston, MA, USA

Jamie T. Twaite Department of Psychology, Queens College and The Graduate Center of the City University of New York, Flushing, NY, USA

Gary Tye Neurosurgery, Virginia Commonwealth University, Richmond, VA, USA 
Katherine Tyson Department of Psychology, University of Connecticut, Storrs, CT, USA

Michelle Uher Marketing Communications, American Academy of Neurology, Minneapolis, MN, USA

Jason Van Allen Clinical Psychology Program, Texas Tech University, Lubbock, TX, USA

Faye van der Fluit Department of Psychology, University of WisconsinMilwaukee, Milwaukee, WI, USA

Timothy E. Van Meter Neurosurgery, Virginia Commonwealth University, Richmond, VA, USA

Gertina J. van Schalkwyk Department of Psychology, University of Macau, Taipa, Macao (SAR), China

Emily Vanderbleek Department of Psychology, University of Notre Dame, Notre Dame, IN, USA

Susan Vandermorris Neuropsychology and Cognitive Health Program, Baycrest, Toronto, ON, USA

Kimberly J. Vannest Department of Educational Psychology, Texas A\&M University, College Station, TX, USA

Jamie Vannice Applied Psychology and Counselor Education, University of Northern Colorado, Greeley, CO, USA

Laura M. Vasel Department of Educational Psychology, Ball State University, Muncie, IN, USA

Rebecca Vaurio Kennedy Krieger Institute, Baltimore, MD, USA

Jennifer Venegas Department of Educational Psychology, The University of Utah, Salt Lake City, UT, USA

Mieke Verfaellie Memory Disorders Research Center, VA Boston Healthcare System and Boston University School of Medicine, Boston, MA, USA

Elizabeth K. Vernon Department of Psychology, Utah State University, Logan, UT, USA

Jean Vettel US Army Research Laboratory, National Academy of the Sciences, Washington, DC, USA

Clara Vila-Castelar Department of Psychology, Queens College and The Graduate Center, The City University of New York, New York, NY, USA

Michael R. Villanueva Department of Psychology, University of North Carolina at Charlotte, Charlotte, NC, USA

Martin A. Volker School and Educational Psychology, University at Buffalo, The State University of New York, Buffalo, NY, USA 
Fred R. Volkmar Child Study Center, Child Psychiatry, Pediatrics and Psychology, Yale University School of Medicine, New Haven, CT, USA

Scott Vota Department of Neurology, Virginia Commonwealth University, Richmond, VA, USA

Christopher Wagner Department of Rehabilitation Counseling, Virginia Commonwealth University, Richmond, VA, USA

George C. Wagner Department of Psychology, Rutgers University, New Brunswick, NJ, USA

Natalie Wahmhoff Department of Educational Psychology, The University of Utah, Salt Lake City, UT, USA

Sarah E. Wallace John G. Rangos Sr. School of Health Sciences, Department of Speech-Language Pathology, Duquesne University, Pittsburgh, PA, USA

Erin Walsh Department of Pharmacology, Virginia Commonwealth University, Richmond, VA, USA

James Walsh School of Psychology, The Chicago School of Professional Psychology, Chicago, IL, USA

Julie L. Wambaugh Veterans Affairs Salt Lake City Healthcare System, University of Utah, Salt Lake City, UT, USA

Yuan Yuan Wang Faculty of Health Sciences, University of Macau, Macao, China

Adam B. Warshowsky Clinical Neuropsychologist, Dual/SCI Unit, Mount Sinai Medical Center, Shepherd Center, Atlanta, GA, USA

Seth Warschausky Department of Physical Medicine and Rehabilitation, University of Michigan, Ann Arbor, MI, USA

David Watson Department of Psychology, 124B Haggar Hall, University of Notre Dame, Notre Dame, IN, USA

Nadia Webb Children's Hospital of New Orleans, New Orleans, LA, USA

Victoria L. Webb Department of Behavioral Sciences, Rush University Medical Center, Chicago, IL, USA

Erica Weber Department of Physical Medicine and Rehabilitation, Kessler Foundation, East Hanover, NJ, USA

Stephen T. Wegener Division of Rehabilitation Psychology and Neuropsychology Department of Physical Medicine and Rehabilitation, The Johns Hopkins School of Medicine, Baltimore, MD, USA

Alan Weintraub Craig Hospital, Rocky Mountain Regional Brain Injury System, Englewood, CO, USA

Devon H. Weir Department of Psychology, University of Florida, Gainesville, FL, USA 
Jonathan Wellman St. Luke's Hospital, Kansas City, MO, USA

John D. Westbrook National Center for the Dissemination of Disability Research (NCDDR), SEDL, Austin, TX, USA

Michael Westerveld Medical Psychology Associates, Florida Hospital, Orlando, FL, USA

Holly James Westervelt Memory and Cognitive Assessment Program, Department of Psychiatry, Rhode Island Hospital, Providence, RI, USA

Kristine B. Whigham Department of Neuropsychology, Children's Healthcare of Atlanta, Atlanta, GA, USA

Gale G. Whiteneck Craig Hospital, Englewood, CO, USA

John Whyte Moss Rehabilitation Research Institute, Albert Einstein Healthcare Network, Elkins Park, PA, USA

Robert G. Will University of Edinburgh, Edinburgh, UK

Gavin Williams Epworth Rehabilitation Centre Epworth Hospital, Richmond Melbourne, VIC, Australia

Travis Williams Department of Physical Medicine and Rehabilitation, Indiana University, Indianapolis, IN, USA

Tricia S. Williams Division of Neurology and Department of Psychology, Sick Kids-Centre for Brain and Mental Health, Toronto, ON, Canada

John B. Williamson Department of Neurology, University of Florida College of Medicine, Center for Neurological Studies and the Research Service of the Malcom Randall Veterans Affairs Medical Center, Gainesville, FL, USA

Meredith L. C. Williamson Department of Primary Care Medicine, College of Medicine, Texas A\&M Health Science Center, Bryan, TX, USA

Brenda Wilson Department of Communication Disorders and Sciences, Eastern Illinois University, Charleston, IL, USA

Jill Winegardner Oliver Zangwill Centre, Ely, UK

Melissa Wingate Neuropsychology, The Chicago School of Professional Psychology, Chicago, IL, USA

Deborah Witsken University of Minnesota Medical School, Minneapolis, MN, USA

Ericka L. Wodka Center for Autism and Related Disorders, Kennedy Krieger Institute and The Johns Hopkins University School of Medicine, Baltimore, MD, USA

Thomas R. Wodushek School of Medicine, Department of Neurosurgery, University of Colorado, Aurora, CO, USA

Edison Wong Center for Pain and Medical Rehabilitation, Fitchburg, MA, USA 
Sarah Woodrow Oak Grove School District, San Jose, CA, USA

Adam J. Woods Department of Clinical and Health Psychology, College of Public Health and Health Professions, University of Florida, Gainesville, FL, USA

Center for Cognitive Aging and Memory, McKnight Brain Institute, University of Florida, Gainesville, FL, USA

Department of Neuroscience, University of Florida, Gainesville, FL, USA

Michael S. Worden Department of Neuroscience, Brown University, Providence, RI, USA

Jerry Wright Rehabilitation Research Center, Santa Clara Valley Medical Center, San Jose, CA, USA

Fan Wu Outcomes Management, The Harris Center for Mental Health and IDD, Houston, TX, USA

Glenn Wylie Neuropsychology and Neuroscience Laboratory, Kessler Medical Rehabilitation Research and Education Center Kessler Foundation, West Orange, NJ, USA

Naohide Yamamoto Queensland University of Technology, Brisbane, Queensland, Australia

Keith Owen Yeates Department of Psychology, University of Calgary, Calgary, AB, Canada

Angela Yi Department of Rehab Medicine, Mount Sinai School of Medicine, New York, NY, USA

Brian Yochim Department of Psychology, University of Colorado at Colorado Springs, Colorado Springs, CO, USA

Sevilay Yumusak Department of Psychology, University of Florida, Gainesville, FL, USA

Michele L. Zaccario Department of Psychology, Pace University, Dyson College of Arts and Sciences, New York, NY, USA

Christina Zafiris Applied Psychology and Counselor Education, University of Northern Colorado, Greeley, CO, USA

Ross Zafonte Department of Physical Medicine and Rehabilitation, Spaulding Rehabilitation Hospital, Massachusetts General Hospital, Brigham and Women's Hospital, Harvard Medical School, Boston, MA, USA

Nathan D. Zasler Concussion Care Centre of Virginia, Ltd., Richmond, VA, USA

Islam Zaydan Neurology, Virginia Commonwealth University, Richmond, VA, USA

Fadel Zeidan Department of Neurobiology and Anatomy, Wake Forest School of Medicine, Winston-Salem, NC, USA 
J. Zhou School Psychology, The Chicago School of Professional Psychology, Chicago, IL, USA

Zheng Zhou Department of Psychology, St. John's University, Queens, NY, USA

Miriam Zichlin Health Economics and Outcomes Research, Analysis Group, Inc., Boston, MA, USA

Rosemary Ziemnik Department of Psychology, The University of Utah, Salt Lake City, UT, USA

Molly E. Zimmerman Department of Psychology, Fordham University, Bronx, NY, USA

Davor Zink Neuropsychology Research Program, University of Nevada, Las Vegas, Las Vegas, NV, USA 